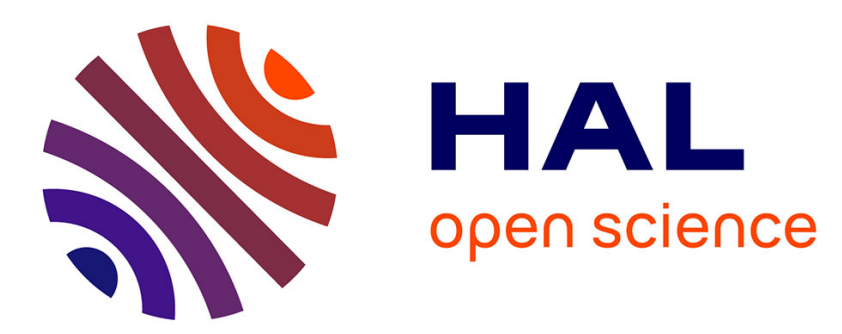

\title{
Parameter selection in a Mumford-Shah geometrical model for the detection of thin structures
}

\author{
Maïtine Bergounioux, David Vicente
}

\section{To cite this version:}

Maïtine Bergounioux, David Vicente. Parameter selection in a Mumford-Shah geometrical model for the detection of thin structures. Acta Applicandae Mathematicae, 2015, pp xx. 10.1007/s10440-0140002-1. hal-00918723v2

\section{HAL Id: hal-00918723 \\ https://hal.science/hal-00918723v2}

Submitted on 7 Jul 2014

HAL is a multi-disciplinary open access archive for the deposit and dissemination of scientific research documents, whether they are published or not. The documents may come from teaching and research institutions in France or abroad, or from public or private research centers.
L'archive ouverte pluridisciplinaire HAL, est destinée au dépôt et à la diffusion de documents scientifiques de niveau recherche, publiés ou non, émanant des établissements d'enseignement et de recherche français ou étrangers, des laboratoires publics ou privés. 


\title{
PARAMETER SELECTION IN A MUMFORD-SHAH GEOMETRICAL MODEL FOR THE DETECTION OF THIN STRUCTURES
}

\author{
MAÏTINE BERGOUNIOUX \& DAVID VICENTE
}

\begin{abstract}
We present a variational model to perform the segmentation of thin structures in MRI images (namely codimension 1 objects). It is based on the classical Mumford-Shah functional and we have added geometrical priors as constraints. We precisely describe the structure model (that we call tubes) and write the problem as a bilevel problem. We focus on the lower level optimization problem and give existence, uniqueness and regularity results for the solution. The keypoint is the fact that $2 \mathrm{D} / 3 \mathrm{D}$ problems are equivalent to $1 \mathrm{D}$ ones. This gives hints to perform an automatic parameter tuning for numerical purpose.
\end{abstract}

\section{INTRODUCTION}

The detection of blood vessels and the complete reconstruction of the network is one of the most challenging problems in biological image processing. Some angiography images are not very noisy and the identification of the network can be done by classical methods that we mention below. However, in some cases, the images are very noisy and undersampled. This is the case for example of angiographic MRI brain network mouse ${ }^{1}$. Even if the magnetic fields are high, the images are undersampled and low contrasted due to the smallness of the observed area. On the other hand the nature of the structure to identify (filaments of codimension 1) requires the development of models to identify objects of null measure.

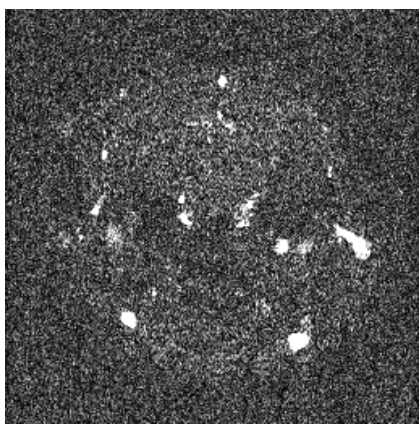

(a) $2 \mathrm{D}$ slice $(256 \times 256)$

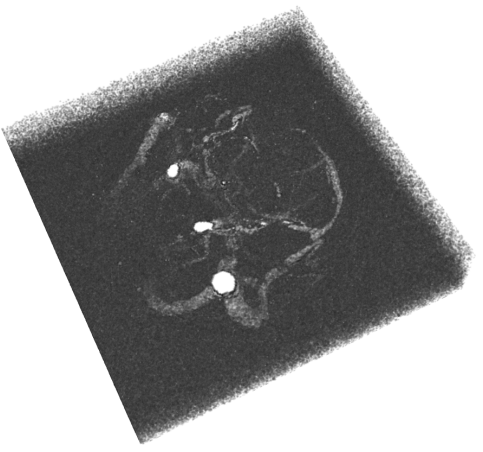

(b) $3 \mathrm{D}$ view (54 slices)

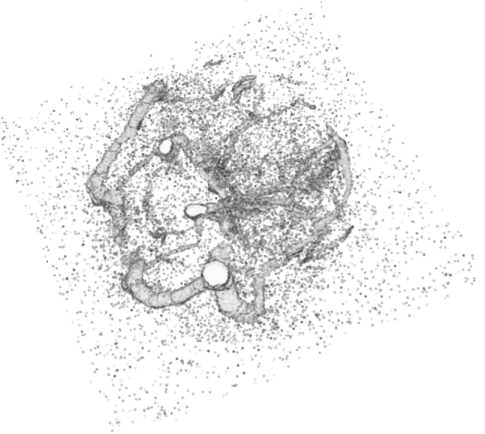

(c) $3 \mathrm{D}$ view (54 slices)

FiguRE 1.1. Mouse brain MRI image with manual threshold

Several approaches have been made to overcome this difficulty both from the point of view in the theoretical aspect (models) and numerics (how to approach and/or discretize such structures). There are, to our knowledge, few models providing a satisfactory answer to these problems. In [15] many vessel extraction techniques and algorithms are

Date: July 7, 2014.

${ }^{1}$ We thank the laboratory CBM in Orléans for the images 
presented. Vessel segmentation algorithms and techniques are divided into six main categories: pattern recognition techniques, model-based, tracking-based , artificial intelligencebased, neural network-based and tube-like object detection approaches. One can find a review of 3D vessel segmentation techniques in [17]. Recently, Péchaud et al. [21] have presented a method to extract a network of vessels centerlines from a medical image. They use both geodesic based methods and tracking methods in a 4D framework. Rouchdy and Cohen [23] use a geodesic voting method to consider the problem. In this paper, we have decided to use a variational model. Such models have been investigated by Aubert and al $[7,8,9,10,14]$ especially in the detection of points in $2 \mathrm{D}$ images. One important tool is the capacity theory [1].

We present here a modified Mumford-Shah model. This model [20, 19] is a well known segmentation model whose approximation has been studied by many people (see for example [12, 13, 18, 16, 22]. We use the Ambrosio-Tortorelli [4] approach and consider an approximate model that $\Gamma$ - converges to the original one. As in [5] we add prior information on the objects but we involve this prior in the constraints rather than in the cost functional.

The paper is organized as follows. We first present the exact model we use and the approximated one. However, these models are not convex and we have a lack of uniqueness. Therefore we consider geometrical constraints in the objects that have to be what we define as tubes of small diameter. Section 3 is devoted to the description of $2 \mathrm{D}$ and $3 \mathrm{D}$ tubes and write the original problem as a two level minimization problem. We focus on the low-level. In the last section we prove that the problem reduces to a $1 \mathrm{D}$ problem and we give some qualitative properties of the (unique) solution. This allows to get an automatic parameter selection: indeed variational models are efficient but the parameter tuning is a major challenge.

\section{A Mumford- Shah type Model}

2.1. The exact model. Let $\Omega$ be an open bounded subset of $\mathbb{R}^{N} \quad(N=2,3)$ smooth enough (with the cone property and $\mathcal{C}^{1}$ for example). Let be $g: \Omega \rightarrow[0,1]$ the (normalized) observed image. The model we study is derived from the Mumford-Shah one [20] that we briefly recall : we look for a pair $(u, K)$ where $K \subset \Omega$ is the set of discontinuities of $g$ and $u$ is a regular function defined on $\Omega \backslash K$. This representation must minimize the following energy:

$$
\mathcal{E}(u, K)=\frac{1}{2} \int_{\Omega \backslash K}|u-g|^{2} d x+\beta \mathcal{H}^{n-1}(K)+\gamma \int_{\Omega \backslash K}|\nabla u|^{2} d x,
$$

where $\beta, \gamma>0$ and $\mathcal{H}^{N-1}(K)$ is the Hausdorff measure of the $N-1$ dimensional set $K$. The first term is a fitting data term and the second ones penalizes the length (if $N=2$ ) or area (if $N=3$ ) of the discontinuity set. The last term penalizes $u$ variations.

We want to split the image in two sub-domains $A$ and $\Omega \backslash A$. So we only consider binary functions $u=\chi_{A}$ where:

$$
\chi_{A}(x)= \begin{cases}1 & \text { si } x \in A, \\ 0 & \text { si } x \in \Omega \backslash A,\end{cases}
$$

The energy we have to minimize writes :

$$
\mathcal{E}\left(\chi_{A}, \partial A\right)=\frac{1}{2} \int_{\Omega}\left|\chi_{A}-g\right|^{2} d x+\beta \mathcal{H}^{n-1}(\partial A) .
$$

To describe the jumps of the function $u$, the most suitable space is the space of of functions with bounded variation $B V(\Omega)$. We recall the definition and the main properties of this space (see $[3,6,11]$ for example), defined by

$$
B V(\Omega)=\left\{u \in L^{1}(\Omega) \mid \Phi_{1}(u)<+\infty\right\},
$$


where

$$
\Phi_{1}(u):=\sup \left\{\int_{\Omega} u(x) \operatorname{div} \xi(x) d x \mid \xi \in \mathcal{C}_{c}^{1}(\Omega),\|\xi\|_{\infty} \leq 1\right\} .
$$

The space $B V(\Omega)$, endowed with the norm $\|u\|_{B V(\Omega)}=\|u\|_{L^{1}}+\Phi_{1}(u)$, is a Banach space. The derivative in the sense of distributions of every $u \in B V(\Omega)$ is a bounded Radon measure, denoted $D u$, and $\Phi_{1}(u)=\int_{\Omega}|D u|$ is the total variation of $u$. We next recall standard properties of functions of bounded variation .

Proposition 2.1. Let $\Omega$ be an open subset of $\mathbb{R}^{N}$ with Lipschitz boundary.

(1) For every $u \in B V(\Omega)$, the Radon measure $D u$ can be decomposed into $D u=$ $\nabla u d x+D^{s} u$, where $\nabla u d x$ is the absolutely continuous part of Du with respect of the Lebesgue measure and $D^{s} u$ is the singular part.

(2) The mapping $u \mapsto \Phi_{1}(u)$ is lower semi-continuous from $B V(\Omega)$ to $\mathbb{R}^{+}$for the $L^{1}(\Omega)$ topology.

(3) $B V(\Omega) \subset L^{\sigma}(\Omega)$ with continuous embedding, for $\sigma \in\left[1, \frac{N}{N-1}\right](N \neq 1)$.

(4) $B V(\Omega) \subset L^{\sigma}(\Omega)$ with compact embedding, for $\sigma \in\left[1, \frac{N}{N-1}\right)(N \neq 1)$.

For $u \in \mathcal{B}(\Omega)$ a borelian function and $x \in \Omega$, we denote

$$
\begin{aligned}
& u^{+}(x)=\inf \left\{t \in \mathbb{R}: \lim _{r \rightarrow 0^{+}} \frac{\mathcal{L}^{N}(B(x, r) \cap[u>t])}{r^{N}}=0\right\}, \\
& u^{-}(x)=\sup \left\{t \in \mathbb{R}: \lim _{r \rightarrow 0^{+}} \frac{\mathcal{L}^{N}(B(x, r) \cap[u<t])}{r^{N}}=0\right\} .
\end{aligned}
$$

The singular set of $u$ is defined as

$$
S_{u}=\left\{x \in \Omega: u^{-}(x)<u^{+}(x)\right\} .
$$

For any function $p$ with bounded variation which is binary (for example $\chi_{A}$ functions), the singular part of its derivative is included in $S_{p}$.

The problems we finally consider writes

$$
\operatorname{Min}\left\{\frac{1}{2} \int_{\Omega}|p-g|^{2} d x+\beta \mathcal{H}^{N-1}\left(S_{p}\right): p \in B V(\Omega), p \in\{0,1\} \text { a.e. }\right\} \text {. }
$$

2.2. Approximate model. The study of the Mumford-Shah model is still challenging and it is easier to consider approximate versions. Modica and Tortola ([19]) prove a $\Gamma$-convergence result for functional

$$
F_{\varepsilon}(u)=\int_{\Omega} \varepsilon|\nabla u|^{2}+\frac{W(u)}{\varepsilon}
$$

to the area functional for surface of dimension $N-1$. Inspired by this work, we set

$$
\mathcal{E}_{\varepsilon}(p)=\frac{1}{2} \int_{\Omega}(p-g)^{2}+\beta \int_{\Omega} 9 \varepsilon|\nabla p|^{2}+\frac{p^{2}(1-p)^{2}}{\varepsilon},
$$

and define the approximate problem as

$$
\min \left\{\mathcal{E}_{\varepsilon}(p) \mid p \in H^{1}(\Omega), 0 \leq p \leq 1 \text { a.e. }\right\} .
$$

The proof is standard but it has been detailed in this very case for the convenience of the reader in [24]. It is easy to prove that $\left(\mathcal{P}_{\varepsilon}\right)$ has at least an optimal solution $p_{\varepsilon}$. However, as $\mathcal{E}_{\varepsilon}$ is not convex, we get no uniqueness.

Problem $\left(\mathcal{P}_{\varepsilon}\right)$ is a suitable approximation of $(\mathcal{P})$. Indeed we have the following classical convergence result: 
Theorem 2.1. For every $\varepsilon>0$, let $p_{\varepsilon}$ be a solution to $\left(\mathcal{P}_{\varepsilon}\right)$. Then we may extract a subsequence $p_{\varepsilon_{n}}$ that converges a.e. to a binary function $\bar{p}(\bar{p}(x) \in\{0,1\}$ a.e. $x)$ which is a solution to $(\mathcal{P})$.

We refer to [6] for definition of the $\Gamma$-convergence and related properties.

\section{Tube Detection}

The functional $\mathcal{E}_{\varepsilon}$ is not convex because of the term $p \rightarrow \int_{\Omega} \frac{\left(p-p^{2}\right)^{2}}{\varepsilon} d x$. Therefore we cannot ensure the uniqueness of the solution to $\left(\mathcal{P}_{\varepsilon}\right)$. As we get existence however, we must refine the model adding a geometrical prior. So we consider the minimization of $\mathcal{E}_{\varepsilon}$ on the set of tubes (that we are going to define). This will provide a unique minimizer according to the tube geometry.

3.1. Modeling a tube. We present here a description of what we call (thin) tubes both for the $2 \mathrm{D}$ and $3 \mathrm{D}$ dimension. Roughly speaking, we define a tube as a symmetric object of codimension 1 whose length $\ell$ is much greater that the diameter $\alpha$. Let $\Gamma$ be a parametrized curve in $\Omega$ : we get the tube by thickening the curve to get a symmetric object of diameter $\alpha>0$.

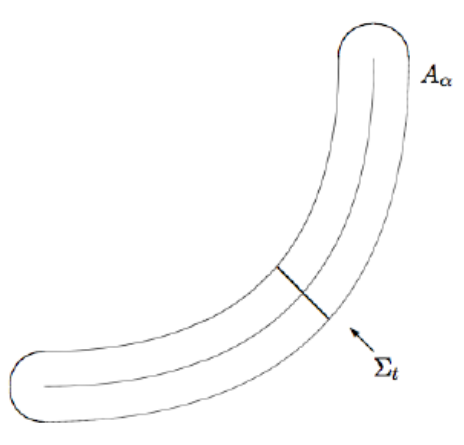

(a) Dimension 2

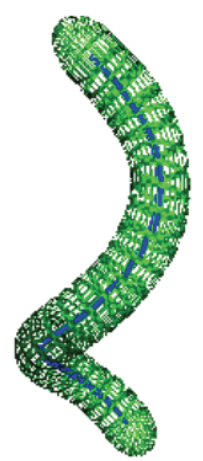

(b) Dimension 3

Figure 3.1. Tubes $A_{\alpha}$

Let us detail the $3 \mathrm{D}$ representation of such tubes. The $2 \mathrm{D}$ case is straightforward (deleting one dimension).

3.1.1. The parametrized curve $\Gamma$. Let $\Gamma \subset \Omega$ be a $\mathcal{C}^{2}$ curve in $\Omega \subset \mathbb{R}^{3}$. We use a parametrization with a curvilinear abscissa $F:[0, \ell] \rightarrow \Gamma$ and assume the following regularity condition :

$$
\left(\mathcal{H}_{\Gamma}\right)\left\{\begin{array}{l}
F \text { is surjective, } \\
F \text { is } \mathcal{C}^{2} \text { and } \forall t \in[0, \ell],\left|F^{\prime}(t)\right|=1, \\
F \text { is biregular }: \forall t \in[0, \ell], \quad \operatorname{dim} \operatorname{Span}\left(F^{\prime}(t), F^{\prime \prime}(t)\right)=2,
\end{array}\right.
$$

where $F^{\prime}(t)$ is the first derivative, $F^{\prime \prime}(t)$ the second derivative and $\langle\cdot, \cdot\rangle$ the $\mathbb{R}^{N}$ inner product. Assumption $\left(\mathcal{H}_{\Gamma}\right)$ allows to define a Frenet-Serret frame whose main properties are recalled thereafter:

Proposition 3.1. Let $\mathbf{S}^{2}$ be the unit sphere of $\mathbb{R}^{3}$ and assume $\left(\mathcal{H}_{\Gamma}\right)$ is fulfilled, then there exist 
- $T:[0, \ell] \rightarrow \mathbf{S}^{2}$ the unit vector tangent to the curve, pointing in the direction of motion,

- $N:[0, \ell] \rightarrow \mathbf{S}^{2}$ the normal unit vector, the derivative of $T$ with respect to the arclength parameter of the curve, divided by its length.,

- $B:[0, \ell] \rightarrow \mathbf{S}^{2}$ the binormal unit vector, the cross product of $T$ and $N$.

Moreover

$$
\frac{d}{d t}\left(\begin{array}{c}
T \\
N \\
B
\end{array}\right)=\left(\begin{array}{ccc}
0 & \gamma & 0 \\
-\gamma & 0 & \tau \\
0 & -\tau & 0
\end{array}\right)\left(\begin{array}{c}
T \\
N \\
B
\end{array}\right) .
$$

Functions $\gamma$ (curvature) and $\tau$ (torsion) are scalar functions and $\gamma$ is nonnegative. The curvature $\gamma$ is the curvature radius inverse.

Remark 3.1. In the $2 D$ case the Frenet-Serret frame reduces to $T$ and $N$. Existence conditions are the same and the differential characterization of the frame is

$$
\frac{d}{d t}\left(\begin{array}{c}
T \\
N
\end{array}\right)=\left(\begin{array}{cc}
0 & \gamma \\
-\gamma & 0
\end{array}\right)\left(\begin{array}{c}
T \\
N
\end{array}\right)
$$

Eventually, we have to set an additional hypothesis to get a local parametrization in the neighborhood of $\Gamma$ : we need the curvature radius to be large enough. If it was smaller than the diameter of the tube, this would correspond to the case where the tube fall back on itself. Therefore, we assume

$$
\forall t \in[0, \ell] \quad \alpha<\inf \left\{\frac{1}{\gamma(t)}\right\} .
$$

Remark 3.2. It is sufficient to assume there exists $\rho>0$ such that

$$
\forall t \in[0, \ell] \quad \frac{\alpha}{2}+\rho<\inf \frac{1}{\gamma(t)} .
$$

We chose $\rho=\frac{\alpha}{2}$ for the sake of simplicity

We may now define the tube $A_{\alpha}$ with thickness $\alpha$ around $\Gamma$ as

$$
A_{\alpha}=\{x \in \Omega: \mathbf{d}(x, \Gamma)<\alpha / 2\} \text { and } g=\chi_{A_{\alpha}}=\left\{\begin{array}{lc}
1 & \text { on } A_{\alpha} \\
0 & \text { elsewhere. }
\end{array}\right.
$$

Here $\mathbf{d}$ is the euclidean distance in $\mathbb{R}^{N}$. Let us divide $A_{\alpha}$ into three sub-areas: the two ends $B_{\alpha}^{0}, B_{\alpha}^{\ell}$ and the body $C_{\alpha}$. More precisely

$$
\begin{aligned}
& B_{\alpha}^{0}=\left\{x \in A_{\alpha}:\|x-F(0)\|=\mathbf{d}(x, \Gamma)\right\}, \\
& B_{\alpha}^{\ell}=\left\{x \in A_{\alpha}:\|x-F(\ell)\|=\mathbf{d}(x, \Gamma)\right\} .
\end{aligned}
$$

and $C_{\alpha}=A_{\alpha} \backslash\left(B_{\alpha}^{0} \cup B_{\alpha}^{\ell}\right)$.

3.1.2. Parametrization of the tube. In order to perform calculations, we must specify the tube parametrization. For this, we consider the ends and the body separately and use spherical coordinates (or polar coordinates for the 2D case).

Proposition 3.2. Assume $\left(\mathcal{H}_{\Gamma}\right)$ and (3.3). Then we may define

- $2 D$ case $(N=2)$ :

$$
\begin{aligned}
\left.\Phi_{C}:[0, \ell] \times\right]-\frac{\alpha}{2}, \frac{\alpha}{2}[ & \rightarrow C_{\alpha} \\
(t, r) & \mapsto F(t)+r N(t),
\end{aligned}
$$




$$
\begin{aligned}
\left.\Phi_{B^{0}}:\right] 0, \frac{\alpha}{2}[\times] 0, \pi[ & \rightarrow B_{\alpha}^{0} \\
(r, \theta) & \mapsto F(0)+r \cos (\theta) N(0)-r \sin (\theta) T(0), \\
\left.\Phi_{B^{\ell}}:\right] 0, \frac{\alpha}{2}[\times] 0, \pi[ & \rightarrow B_{\alpha}^{\ell} \\
(r, \theta) & \mapsto F(\ell)+r \cos (\theta) N(\ell)+r \sin (\theta) T(\ell),
\end{aligned}
$$

- $3 D$ case $(N=3)$ :

$$
\begin{gathered}
\left.\Phi_{C} \quad: \quad[0, \ell] \times\right]-\frac{\alpha}{2}, \frac{\alpha}{2}[\times]-\frac{\pi}{2}, \frac{\pi}{2}\left[\rightarrow C_{\alpha}\right. \\
(t, r, \theta)) \quad \mapsto \quad F(t)+r \cos (\theta) N(t)+r \sin (\theta) B(t) . \\
\left.\Phi_{B^{0}}: \quad\right] 0, \frac{\alpha}{2}[\times] 0,2 \pi[\times] 0, \frac{\pi}{2}\left[\rightarrow B_{\alpha}^{0}\right. \\
(r, \theta, \phi) \quad \mapsto \quad F(0)+r \cos (\phi)(\cos (\theta) N(0)+\sin (\theta) B(0))-r \sin (\phi) T(0), \\
\left.\Phi_{B^{\ell}}: \quad\right] 0, \frac{\alpha}{2}[\times] 0,2 \pi[\times] 0, \frac{\pi}{2}\left[\rightarrow B_{\alpha}^{\ell}\right. \\
(r, \theta, \phi) \quad \mapsto \quad F(\ell)+r \cos (\phi)(\cos (\theta) N(\ell)+\sin (\theta) B(\ell))+r \sin (\phi) T(\ell),
\end{gathered}
$$

Moreover $\Phi_{C}$ is a local diffeomorphism whose jacobian is

$$
\begin{aligned}
& J \Phi_{C}(t, r)=\quad 1-r \gamma(t) \quad \text { if } N=2 \text {, } \\
& J \Phi_{C}(t, r, \theta)=r(1-r \cos (\theta) \gamma(t)) \text { if } N=3 \text {. }
\end{aligned}
$$

Proof. Using the 3D Frenet-Serret formulas (3.1) gives

$$
J \Phi_{C}(t, r, \theta)=\left|\begin{array}{ccc}
1-\gamma(t) r \cos \theta & 0 & 0 \\
-\tau(t) r \sin \theta & \cos \theta & -r \sin \theta \\
\tau(t) r \cos \theta & \sin \theta & r \cos \theta
\end{array}\right|=r(1-r \cos (\theta) \gamma(t)) .
$$

As $r<\alpha / 2$ and the curvature radius of $\Gamma$ is always greater than $\alpha / 2$ so that

$$
\forall t \in[0, \ell] \quad \mid 1-r \cos (\theta) \gamma(t)) \mid \neq 0 .
$$

This proves that $\Phi_{C}$ is a local diffeomorphism.

Now, we gather the respective parametrizations $\Phi_{B^{0}}, \Phi_{B^{\ell}}$ and $\Phi_{C}$ to get only one denoted $\Phi$ defined on $A_{\alpha}$.

Definition 3.1. Assume $\left(\mathcal{H}_{\Gamma}\right)$ and (3.3). We say that $\left(A_{\alpha}, \Gamma\right)$ is a tube if the application $\Phi$ is a $\mathcal{C}^{1}$ global diffeomorphism.

3.1.3. The set $\mathcal{F}_{\alpha}$ of tubes of diameter $\alpha$. From now we assume that $\left(\Gamma, A_{\alpha}\right)$ is a tube (as in definition 3.1). We now define a set of feasible functions to describe such a tube.

Definition 3.2. Let $\left(\Gamma, A_{\alpha}\right)$ be a tube satisfying $\left(\mathcal{H}_{\Gamma}\right)$ and (3.3). The space $\mathcal{F}_{\alpha} \subset H_{0}^{1}(\Omega)$ is defined as the space of $H_{0}^{1}(\Omega)$ functions $p$ such that

a) for almost every $x \in \Omega \backslash A_{\alpha}, p(x)=0$,

b) for almost every $(x, \tilde{x}) \in A_{\alpha} \times A_{\alpha}$,

$$
\mathbf{d}(x, \Gamma)=\mathbf{d}(\tilde{x}, \Gamma) \Rightarrow p(x)=p(\tilde{x}) .
$$

Remark 3.3. According the thickness of tubes, we can assume that a functional $p$ which is a solution of our problem is almost equal to 0 outside the tubes. 
These conditions mean that $p$ has its support in $A_{\alpha}$ and is symmetric with respect to the center $\Gamma$ of the tube.

We end with an injectivity property of $\mathcal{F}_{\alpha}$ functions.

Lemma 3.1. Let $\left(\Gamma, A_{\alpha}\right)$ be a tube satisfying $\left(\mathcal{H}_{\Gamma}\right)$ and (3.3). Then, for $p \in \mathcal{F}_{\alpha}$, for almost every $(t, \tilde{t}) \in[0, \ell]^{2},(\theta, \tilde{\theta}) \in(]-\frac{\pi}{2}, \frac{\pi}{2}[)^{2}$ and $(r, \tilde{r}) \in(]-\frac{\alpha}{2}, \frac{\alpha}{2}[)^{2}$, we get:

$$
|r|=|\tilde{r}| \Rightarrow p(\Phi(t, r, \theta))=p(\Phi(\tilde{t}, \tilde{r}, \tilde{\theta})) .
$$

Proof. • Let be $t \in] 0, \ell[,(r, \theta) \in]-\frac{\alpha}{2}, \frac{\alpha}{2}[\times]-\frac{\pi}{2}, \frac{\pi}{2}\left[\right.$ and set $x=\Phi_{C}(t, r, \theta)$. Let $y \in \Gamma$ be a point that minimizes the distance of $x$ to $\Gamma$ (it exists since $\Gamma$ is compact). Let $\left.t_{0} \in\right] 0, \ell\left[\right.$ be such that $y=F\left(t_{0}\right)$. As $\Gamma$ is smooth, $x-F\left(t_{0}\right)$ is a normal vector to $\Gamma$ at $F\left(t_{0}\right)$. As the tangent plane is the affine plane $F\left(t_{0}\right)+\operatorname{Span}\left(N\left(t_{0}\right), B\left(t_{0}\right)\right)$, there exists $\left.\left(r_{0}, \theta_{0}\right) \in\right]-\frac{\alpha}{2}, \frac{\alpha}{2}[\times]-\frac{\pi}{2}, \frac{\pi}{2}[$ such that

$$
x=F\left(t_{0}\right)+r_{0} \cos \left(\theta_{0}\right) N\left(t_{0}\right)+r_{0} \sin \left(\theta_{0}\right) B\left(t_{0}\right) ;
$$

this means that $x=\Phi_{C}\left(t_{0}, r_{0}, \theta_{0}\right)$. With (3.2), $\Phi$ is a global diffeomorphism. This implies that $t=t_{0}, r=r_{0}$ and $\theta= \pm \theta_{0}$. In particular, we may conclude that $\mathbf{d}(\Phi(t, r, \theta), \Gamma)=|r|$. - Let be $(r, \theta, \phi) \in]-\frac{\alpha}{2}, \frac{\alpha}{2}[\times]-\pi, \pi[\times] 0, \frac{\pi}{2}\left[\right.$ and set $x=\Phi_{B^{\ell}}(r, \theta, \phi)$. We prove similarly that $\mathbf{d}(x, \Gamma)=|r|$. The same reasoning holds for $B^{0}$.

- We just proved that

$$
|r|=|\tilde{r}| \Rightarrow \operatorname{dist}(\Phi(t, r, \theta), \Gamma)=\mathbf{d}(\Phi(\tilde{t}, \tilde{r}, \tilde{\theta}), \Gamma) .
$$

With proposition 3.2 , this yields that

$$
|r|=|\tilde{r}| \Rightarrow p(\Phi(t, r, \theta))=p(\Phi(\tilde{t}, \tilde{r}, \tilde{\theta})) .
$$

3.1.4. Minimization problem with tube constraints. We now set the new formulation of the problem which takes into account the tube constraints. We have to consider all the tubes, namely all the curves $\Gamma \in \mathcal{C}^{2}(\Omega)$ that satisfy assumption $\left(\mathcal{H}_{\Gamma}\right)$ and, once $\Gamma$ is fixed the constraint $p \in \mathcal{F}_{\alpha}$ :

$$
\min _{\Gamma \in \mathcal{C}^{2}} \min _{p \in \mathcal{F}_{\alpha}} \mathcal{E}_{\varepsilon}(p)
$$

This problem is difficult to handle because the space $\mathcal{C}^{2}$ is not suitable for variational analysis. There is a great lack of compactness and we are not able to prove an existence result for an optimal tube.

However, once a curve is chosen, its length $\ell$, curvature and more generally geometric features are chosen and appear as parameters in the so called low-level problem (though the formulation is note quite adapted here):

$$
\min _{p \in \mathcal{F}_{\alpha}} \mathcal{E}_{\varepsilon}(p)
$$

where the curve $(F, \ell)$ is fixed from now.

The end of the paper is devoted to existence an uniqueness result of a solution to $\mathcal{P}_{\varepsilon, \alpha}$. In addition, qualitative properties of the solution will provide parameters tuning with respect to the tube diameter $\alpha$ and length $\ell$.

Here

$$
\mathcal{E}_{\varepsilon}(p)=\frac{1}{2} \int_{A_{\alpha}}(p-1)^{2} d x+\beta \int_{A_{\alpha}}\left(9 \varepsilon|\nabla p|^{2}+\frac{(p(p-1))^{2}}{\varepsilon}\right) d x
$$

since $g:=\chi_{A_{\alpha}}$ and functions in $\mathcal{F}_{\alpha}$ have their support in $A_{\alpha}$.

Theorem 3.1 (Existence). Problem $\left(\mathcal{P}_{\varepsilon, \alpha}\right)$ has at least an optimal solution. 
Proof. Let $\left(p_{n}\right)_{n>1}$ be a minimizing sequence. As $p_{n}$ and $\nabla p_{n}$ are bounded in $L^{2}(\Omega)$, the sequence $\left(p_{n}\right)_{n \geq 1}$ is bounded in $H_{0}^{1}(\Omega)$. Moreover $H_{0}^{1}(\Omega)$ is compactly embedded in $L^{4}(\Omega)(N \leq 3$, see [2]). Therefore, one may extract a subsequence (denoted similarly) that weakly converges to $\bar{p}$ in $H_{0}^{1}(\Omega)$ and strongly in $L^{4}(\Omega)$. The lower semi -continuity of $\mathcal{E}_{\varepsilon}$ then gives

$$
\mathcal{E}_{\varepsilon}(\bar{p}) \leq \liminf _{n \rightarrow+\infty} \mathcal{E}_{\varepsilon}\left(p_{n}\right) .
$$

It remains to prove that $\bar{p} \in \mathcal{F}_{\alpha}$. As $H_{0}^{1}(\Omega)$ is compactly embedded in $L^{1}(\Omega)$ the sequence $\left(p_{n}\right)_{n \geq 1}$ converges to $\bar{p}$ almost everywhere (up to a subsequence). The symmetry properties of definition 3.2 properties are kept by taking the limit. This gives $\bar{p} \in \mathcal{F}_{\alpha}$.

In the sequel we set

$$
\forall t \in \mathbb{R} \quad F_{\beta, \varepsilon}(t)=\frac{1}{2}(t-1)^{2}+\frac{\beta}{\varepsilon}\left(t^{2}-t\right)^{2},
$$

and

$$
\forall t \in \mathbb{R} \quad f_{\beta, \varepsilon}(t)=\frac{1}{2} F_{\beta, \varepsilon}^{\prime}(t)=\frac{\beta}{\varepsilon}\left(2 t^{3}-3 t^{2}\right)+\left(\frac{1}{2}+\frac{\beta}{\varepsilon}\right) t-\frac{1}{2} .
$$

Theorem 3.2 (Optimality condition). Let $\bar{p}$ be a solution to $\left(\mathcal{P}_{\varepsilon, \alpha}\right)$. Then $\bar{p} \in \mathcal{F}_{\alpha}$ verifies

$$
\forall \varphi \in \mathcal{F}_{\alpha} \quad \int_{A_{\alpha}}\left(9 \beta \varepsilon \nabla \bar{p}(x) \nabla \varphi(x)+f_{\beta, \varepsilon}(p(x)) \varphi(x)\right) d x=0 .
$$

Proof. A classical computation gives

$$
\begin{aligned}
\forall \varphi \in \mathcal{F}_{\alpha}<\nabla \mathcal{E}_{\varepsilon}(\bar{p}), \varphi> & =\int_{A_{\alpha}}\left(18 \beta \varepsilon \nabla \bar{p}(x) \nabla \varphi(x)+F_{\beta, \varepsilon}^{\prime}(\bar{p}(x)) \varphi(x)\right) d x, \\
& =2 \int_{A_{\alpha}}\left(9 \beta \varepsilon \nabla \bar{p}(x) \nabla \varphi(x)+f_{\beta, \varepsilon}(\bar{p}(x)) \varphi(x)\right) d x .
\end{aligned}
$$

Every solution $\bar{p} \in \mathcal{F}_{\alpha}$ to $\left(\mathcal{P}_{\varepsilon, \alpha}\right)$ satisfies

$$
\forall \varphi \in \mathcal{F}_{\alpha} \quad<\nabla \mathcal{E}_{\varepsilon}, \varphi-\bar{p}>\geq 0,
$$

that is (since $\mathcal{F}_{\alpha}$ is a linear space)

$$
\forall \varphi \in \mathcal{F}_{\alpha} \quad<\nabla \mathcal{E}_{\varepsilon}(\bar{p}), \varphi>=0 .
$$

Theorem 3.3 (Uniqueness). If $\beta \leq \varepsilon$, then problem $\left(\mathcal{P}_{\varepsilon, \alpha}\right)$ has a unique solution.

Proof. Let $p_{1}$ and $p_{2}$ be two solutions of $\left(\mathcal{P}_{\varepsilon, \alpha}\right)$. As $p_{1}$ and $p_{2}$ belong to $\mathcal{F}_{\alpha}$ one may choose $\varphi=p_{1}-p_{2}$ in (3.9): using this equality with $p_{1}$ and $p_{2}$ respectively and subtracting gives

$$
\int_{A_{\alpha}}\left(9 \beta \varepsilon\left|\nabla\left(p_{1}-p_{2}\right)\right|^{2}+\left(f_{\beta, \varepsilon}\left(p_{1}\right)-f_{\beta, \varepsilon}\left(p_{2}\right)\right)\left(p_{1}-p_{2}\right) d x=0 .\right.
$$

As $\left(f_{\beta, \varepsilon}\left(p_{1}\right)-f_{\beta, \varepsilon}\left(p_{2}\right)\right)\left(p_{1}-p_{2}\right)=f_{\beta, \varepsilon}^{\prime}\left(p_{1}+\theta p_{2}\right)\left(p_{1}-p_{2}\right)^{2}$ with $\theta \in[0,1]$, it is sufficient that $f_{\beta, \varepsilon}^{\prime} \geq 0$ to get $p_{1}=p_{2}$.

$$
\forall t \in \mathbb{R} \quad f_{\beta, \varepsilon}^{\prime}(t)=\frac{6 \beta}{\varepsilon} t^{2}-\frac{6 \beta}{\varepsilon} t+\left(\frac{1}{2}+\frac{\beta}{\varepsilon}\right) .
$$

The discriminant is

$$
D=\frac{36 \beta^{2}}{\varepsilon^{2}}-\frac{24 \beta}{\varepsilon}\left(\frac{1}{2}+\frac{\beta}{\varepsilon}\right)=\frac{12 \beta^{2}}{\varepsilon^{2}}-\frac{12 \beta}{\varepsilon}=\frac{12 \beta}{\varepsilon}\left(\frac{\beta}{\varepsilon}-1\right) .
$$

If $\beta \leq \varepsilon$ then $D \leq 0$; this implies that $f_{\beta, \varepsilon}^{\prime} \geq 0$. 
Remark 3.4. We chose the (classical) coefficient $\frac{1}{2}$ for the fitting data term. We may, however, introduce a parameter $\gamma$ to adjust the weights of the different terms. More precisely we can define

$$
\mathcal{E}_{\gamma, \varepsilon}(p)=\frac{\gamma}{2} \int_{A_{\alpha}}(p-1)^{2} d x+\beta \int_{A_{\alpha}}\left(9 \varepsilon|\nabla p|^{2}+\frac{(p(p-1))^{2}}{\varepsilon}\right) d x
$$

where $\gamma>0$. It is easy to see that minimizing $\mathcal{E}_{\gamma, \varepsilon}$ is equivalent to minimizing $\mathcal{E}_{\varepsilon}$ with $\frac{\beta}{\gamma}$ instead of $\beta$. The uniqueness condition writes then $\beta \leq \gamma \varepsilon$.

The first significant result of this section is the existence of a unique solution providing $\beta \leq \varepsilon$ : this is a first rough parameter tuning. More informations come from the optimality conditions that we make precise now. Indeed, the constraint $p \in \mathcal{F}_{\alpha}$ does not go directly to a partial differential equation from (3.9): we cannot ensure that the solution of such an equation (to be computed in the dual of $\mathcal{F}_{\alpha}$ ) exists and belongs to $\mathcal{F}_{\alpha}$. In addition, the numerical description of $\mathcal{F}_{\alpha}$ is difficult. For all these reasons, we first show that the $2 \mathrm{D} / 3 \mathrm{D}$ problem $\left(\mathcal{P}_{\varepsilon, \alpha}\right)$ can be reduced to a problem in one dimension. We can then give specific properties of the solution and provide an automatic selection of parameters $\beta$ and $\varepsilon$ with respect to $\alpha$ et $\ell$.

3.2. Reduction to a one dimensional problem. To reduce the problem $\left(\mathcal{P}_{\varepsilon, \alpha}\right)$ to a $1 \mathrm{D}$ problem, we exhibit a diffeomorphism that allows to fully describe a tube (through its parametrization ) with a single variable. This is made possible by the very specific definition of the concept of tube. We will have to relax some assumptions later to handle the case of more general tubes.

The 1D problem we obtain is formulated in a weighted Sobolev space where the weight $\omega$ is related to the geometry of the tube and (therefore) the space dimension. The case of dimensions 2 and 3 are treated in the same way with a significant difference in 3D since the weight $\omega$ vanishes at 0 .

Assume that $\left(\Gamma, A_{\alpha}\right)$ is a tube as in the definition 3.1. The purpose of this section is to obtain an expression of the energy when restricted to $\mathcal{F}_{\alpha}$. In what follows we set

$$
\omega(r)=\frac{\mathcal{H}^{N-1}\left(\partial A_{r}\right)}{2}
$$

where $N=2,3$ is the space dimension, $\mathcal{H}$ the Hausdorff measure and $\partial A_{r}$ the boundary of the tube $A_{|r|}$ (with length $\ell$ ). A quick computation gives

$$
\omega(r)= \begin{cases}\ell+\pi|r| & \text { if } N=2 \\ \pi \ell|r|+2 \pi r^{2} & \text { if } N=3\end{cases}
$$

Definition 3.3. Let be $I_{\alpha}=\left[-\frac{\alpha}{2}, \frac{\alpha}{2}\right]$. The weighted Sobolev space $H_{\omega}^{1}\left(I_{\alpha}\right)$ is defined as

$$
H_{\omega}^{1}\left(I_{\alpha}\right):=\left\{q \in L^{2}\left(I_{\alpha}\right) \mid \int_{I_{\alpha}}\left(|q|^{2}+\left|q^{\prime}\right|^{2}\right) \omega(r) d r<+\infty\right\}
$$

where $\omega$ is given by (3.10). This space is endowed with the norm

$$
\|q\|_{H_{\omega}^{1}\left(I_{\alpha}\right)}^{2}=\int_{I_{\alpha}}\left(|q|^{2}+\left|q^{\prime}\right|^{2}\right) \omega(r) d r
$$

It is easy to see that $H^{1}\left(I_{\alpha}\right)$ is continuously embedded in $H_{\omega}^{1}\left(I_{\alpha}\right)$ since $\omega$ is bounded on $I_{\alpha}$. The converse embedding is true if $N=2$.

Lemma 3.2. If $N=2$, then $H_{\omega}^{1}\left(I_{\alpha}\right)=H^{1}\left(I_{\alpha}\right)$ and the associated norms are equivalent. 
Proof. As $\ell \leq \omega(r) \leq \ell+\pi \frac{\alpha}{2}$, for every $r \in I_{\alpha}$, we get

$$
\ell\|q\|_{H^{1}\left(I_{\alpha}\right)}^{2} \leq\|q\|_{H_{\omega}^{1}\left(I_{\alpha}\right)}^{2} \leq\left(\ell+\pi \frac{\alpha}{2}\right)\|q\|_{H^{1}\left(I_{\alpha}\right)}^{2} .
$$

This achieves the proof.

If $N=2, H_{\omega}^{1}\left(I_{\alpha}\right)=H^{1}\left(I_{\alpha}\right) \subset \mathcal{C}^{0}\left(I_{\alpha}\right)$ (continuous functions on $I_{\alpha}$ ) and the corresponding functions are defined everywhere. In particular the trace on the boundary makes sense.

In the $3 \mathrm{D}$ case lemma 3.2 is false since $\omega(0)=0$. Therefore functions in $H_{\omega}^{1}\left(I_{\alpha}\right)$ may have a singularity at en 0 . However, we still have a continuity results outside 0 .

Lemma 3.3. When $N=3$, then $H_{\omega}^{1}\left(I_{\alpha}\right) \subset \mathcal{C}^{0}\left(I_{\alpha} \backslash\{0\}\right)$.

Proof. Let be $q \in H_{\omega}^{1}\left(I_{\alpha}\right)$ and $\left.\left.r \in\right] 0, \frac{\alpha}{2}\right]$. Let us prove that $q$ is continuous on $I_{\alpha} \backslash[-r, r]$. Choose $\left.r^{\prime} \in\right] 0, r\left[\right.$ and $\nu$ a $C^{1}\left(I_{\alpha}\right)$ function identically equal to 1 on $I_{\alpha} \backslash[-r, r]$ with support in $I_{\alpha} \backslash\left[-r^{\prime}, r^{\prime}\right]$. The function $\nu q$ belongs to $H^{1}\left(I_{\alpha}\right)$. Indeed,

$$
\begin{aligned}
\int_{I_{\alpha}}\left((\nu q)^{\prime}\right)^{2} d r=\int_{I_{\alpha}}\left(\nu^{\prime} q+\nu q^{\prime}\right)^{2} d r & \leq 2 \int_{I_{\alpha}}\left(\left(\nu^{\prime} q\right)^{2}+\left(\nu q^{\prime}\right)^{2}\right) d r \\
& \leq 2\left\|\nu^{\prime}\right\|_{\infty} \int_{I_{\alpha} \backslash\left[-r^{\prime}, r^{\prime}\right]} q^{2} d r+2\|\nu\|_{\infty} \int_{I_{\alpha} \backslash\left[-r^{\prime}, r^{\prime}\right]}\left(q^{\prime}\right)^{2} d r, \\
& \leq \frac{2\left\|\nu^{\prime}\right\|_{\infty}}{\omega\left(r^{\prime}\right)} \int_{I_{\alpha} \backslash\left[-r^{\prime}, r^{\prime}\right]} \omega q^{2} d r+\frac{2\|\nu\|_{\infty}}{\omega\left(r^{\prime}\right)} \int_{I_{\alpha} \backslash\left[-r^{\prime}, r^{\prime}\right]} \omega\left(q^{\prime}\right)^{2} d r, \\
& \leq \frac{2\left\|\nu^{\prime}\right\|_{\infty}}{\omega\left(r^{\prime}\right)} \int_{I_{\alpha}} \omega q^{2} d r+\frac{2\left\|\nu^{\prime}\right\|_{\infty}}{\omega\left(r^{\prime}\right)} \int_{I_{\alpha}} \omega\left(q^{\prime}\right)^{2} d r, \\
& <+\infty,
\end{aligned}
$$

and

$$
\begin{aligned}
\int_{I_{\alpha}}(\nu q)^{2} d r & \leq\|\nu\|_{\infty}^{2} \int_{I_{\alpha} \backslash\left[-r^{\prime}, r^{\prime}\right]} q^{2} d r \leq \frac{\|\nu\|_{\infty}^{2}}{\omega\left(r^{\prime}\right)} \int_{I_{\alpha} \backslash\left[-r^{\prime}, r^{\prime}\right]} \omega(r) q^{2}(r) d r \\
& \leq \frac{\|\nu\|_{\infty}^{2}}{\omega\left(r^{\prime}\right)} \int_{I_{\alpha}} \omega(r) q^{2}(r) d r<+\infty
\end{aligned}
$$

Thus $\nu q$ is a continuous function on $I_{\alpha}$. As $\nu \equiv 1$ on $I_{\alpha} \backslash[-r, r]$, then $q$ is continuous on $I_{\alpha} \backslash[-r, r]$ for every $r>0$. Therefore $q$ is continuous on $I_{\alpha} \backslash\{0\}$.

We may define $1 \mathrm{D}$ spaces analogous to $H_{0}^{1}(\Omega)$ and $\mathcal{F}_{\alpha}$ :

Definition 3.4. Let $\omega$ be defined by (3.10). The space $H_{\omega, 0}^{1}\left(I_{\alpha}\right)$ is the space of $H_{\omega}^{1}\left(I_{\alpha}\right)$ functions that vanish at $-\frac{\alpha}{2}$ and $\frac{\alpha}{2}$. The space $\mathcal{G}_{\alpha}^{\omega}$ is the space of even functions of $H_{\omega, 0}^{1}\left(I_{\alpha}\right)$.

The correspondence between $2 \mathrm{D} / 3 \mathrm{D}$ case and $1 \mathrm{D}$ case is described in next proposition:

Proposition 3.3. The following application $\Theta$ is an isomorphism from $\mathcal{F}_{\alpha}$ to $\mathcal{G}_{\alpha}^{\omega}$ :

$$
\begin{aligned}
\Theta: \mathcal{F}_{\alpha} & \rightarrow \mathcal{G}_{\alpha}^{\omega} \\
p & \rightarrow\left\{\begin{array}{rll}
q: I_{\alpha} & \rightarrow \mathbb{R} \\
r & \mapsto p\left(\Phi_{C}(0, r)\right),
\end{array}\right.
\end{aligned}
$$

where $\Phi_{C}$ is defined with (3.2). Moreover, if $q=\Theta(p)$ then

$$
\|p\|_{H^{1}(\Omega)}=\|q\|_{H_{\omega}^{1}\left(I_{\alpha}\right)},
$$


Proof. (i) Let us show relation (3.11) for $N=2$. For every $p \in \mathcal{F}_{\alpha}$, we have

$$
\begin{aligned}
\|p\|_{H^{1}(\Omega)}^{2}= & \iint_{\Omega}\left(|p|^{2}+|\nabla p|^{2}\right) d x=\iint_{A_{\alpha}}\left(|p|^{2}+|\nabla p|^{2}\right) d x . \\
\|p\|_{H^{1}(\Omega)}^{2}= & \underbrace{\int_{t=0}^{\ell} \int_{r=-\frac{\alpha}{2}}^{\frac{\alpha}{2}}\left(|p|^{2}+|\nabla p|^{2}\right) \circ \Phi_{C}(t, r)|1-r \gamma(t)| d r d t}_{\text {Body } \mathcal{C}_{\alpha}} \\
& +\underbrace{\int_{\theta=0}^{\pi} \int_{r=0}^{\frac{\alpha}{2}}\left(|p|^{2}+|\nabla p|^{2}\right) \circ \Phi_{B^{0}}(r, \theta) r d r d \theta}_{\text {End B } B^{0}} \\
& +\underbrace{\int_{\theta=0}^{\pi} \int_{r=0}^{\frac{\alpha}{2}}\left(|p|^{2}+|\nabla p|^{2}\right) \circ \Phi_{B^{\ell}}(r, \theta) r d r d \theta}_{\text {End B } B^{\ell}}
\end{aligned}
$$

- Let us estimate

$$
I_{1}:=\int_{t=0}^{\ell} \int_{r=-\frac{\alpha}{2}}^{\frac{\alpha}{2}}\left(|p|^{2}+|\nabla p|^{2}\right) \circ \Phi_{C}(t, r)|1-r \gamma(t)| d r d t .
$$

Assumption (3.3) yields that

$$
\forall r \in I_{\alpha}, \forall t \in[0, \ell] \quad 1-r \gamma(t)>0 .
$$

As $p \in \mathcal{F}_{\alpha}$, it is an even function with respect to $r$. Thus, we get

$$
\begin{aligned}
I_{1}= & \int_{t=0}^{\ell} \int_{r=-\frac{\alpha}{2}}^{0}\left(|p|^{2}+|\nabla p|^{2}\right) \circ \Phi_{C}(t, r)(1-r \gamma(t)) d r d t \\
& +\int_{t=0}^{\ell} \int_{r=0}^{\frac{\alpha}{2}}\left(|p|^{2}+|\nabla p|^{2}\right) \circ \Phi_{C}(t, r)(1+r \gamma(t)) d r d t, \\
I_{1}= & 2 \int_{t=0}^{\ell} \int_{r=0}^{\frac{\alpha}{2}}\left(|p|^{2}+|\nabla p|^{2}\right) \circ \Phi_{C}(t, r) d r d t .
\end{aligned}
$$

Thanks to definition 3.2, we get

$$
\forall(t, r) \in[0, \ell] \times I_{\alpha} \quad p\left(\Phi_{C}(t, r)\right)=p\left(\Phi_{C}(0, r)\right) .
$$

Differentiating with respect to $t$, gives

$$
\forall r \in I_{\alpha}, \quad(1-r \gamma(t))\left\langle\nabla p\left(\Phi_{C}(t, r)\right), T(t)\right\rangle=0 .
$$

As $1-r \gamma(t)>0$ then $\nabla p\left(\Phi_{C}(t, \cdot)\right)$ is orthogonal to $T(t)$ and, thus, colinear to $N(t)$. This gives

$$
|\nabla p|^{2} \circ \Phi_{C}(t, r)=\left|\left\langle\nabla p\left(\Phi_{C}(t, r)\right), N(t)\right\rangle\right|^{2} .
$$

Since $q(r)=p(F(t)+r N(t))$ then $q^{\prime}(r)=\left\langle\nabla p\left(\Phi_{C}(t, r)\right), N(t)\right\rangle$.

We finally obtain $q^{\prime}(r)^{2}=|\nabla p|^{2} \circ \Phi_{C}(t, r)$ and

$$
I_{1}=2 \ell \int_{r=0}^{\frac{\alpha}{2}}\left(|q(r)|^{2}+\left|q^{\prime}(r)\right|^{2}\right) d r .
$$

- We notice that $\Phi_{B^{0}}(r, \theta)=\Phi_{B^{0}}(r, 0)$, so that

$$
I_{2}=\int_{\theta=0}^{\pi} \int_{r=0}^{\frac{\alpha}{2}}\left(|p|^{2}+|\nabla p|^{2}\right) \Phi_{B^{0}}(r, \theta) r d r d \theta,
$$


writes

$$
I_{2}=\int_{\theta=0}^{\pi} \int_{r=0}^{\frac{\alpha}{2}}\left(|p|^{2}+|\nabla p|^{2}\right) \Phi_{B^{0}}(r, 0) r d r d \theta=\pi \int_{r=0}^{\frac{\alpha}{2}}\left(|p|^{2}+|\nabla p|^{2}\right) \Phi_{B^{0}}(r, 0) r d r d \theta .
$$

As $\Phi_{B^{0}}(r, \theta)=F(0)+r \cos (\theta) N(0)-r \sin (\theta) T(0)=r$, the function $\theta \rightarrow p(F(0)+$ $r \cos (\theta) N(0)-r \sin (\theta))$ is constant on $[0, \pi]$. The differentiation gives

$$
\forall \theta \in[0, \pi] \quad\left\langle\nabla p \circ \Phi_{B^{0}}(r, \theta),(-\sin (\theta) N(0)-\cos (\theta) T(0))\right\rangle=0 .
$$

This means that $\nabla p \circ \Phi_{B^{0}}(r, \theta)$ is orthogonal to $-\sin (\theta) N(0)-\cos (\theta) T(0)$ and colinear to $\cos (\theta) N(0)-\sin (\theta) T(0)$. In addition, $q(r)=p \circ \Phi_{B^{0}}(r, \theta)$, which yields

$$
\left|q^{\prime}(r)\right|=\left|\left\langle\nabla \nabla p \circ \Phi_{B^{0}}(r, \theta), \cos (\theta) N(0)-\sin (\theta) T(0)\right\rangle\right| \text {. }
$$

As $\cos (\theta) N(0)-\sin (\theta) T(0)$ is a unit vector we get

$$
\left|q^{\prime}(r)\right|=\mid \nabla p(F(0)+r \cos (\theta) N(0)-r \sin (\theta) T(0) \mid .
$$

Eventually

$$
I_{2}=\pi \int_{r=0}^{\frac{\alpha}{2}}\left(|q|^{2}+\left|q^{\prime}\right|^{2}\right) r d r .
$$

- We can prove similarly that

$$
I_{3}:=\int_{\theta=0}^{\pi} \int_{r=0}^{\frac{\alpha}{2}}\left(|p|^{2}+|\nabla p|^{2}\right) \circ \Phi_{B^{\ell}}(r, \theta) r d r d \theta
$$

verifies

$$
I_{3}=\pi \int_{r=0}^{\frac{\alpha}{2}}\left(|q|^{2}+\left|q^{\prime}\right|^{2}\right) r d r .
$$

- The above estimates give

$$
\|p\|_{H^{1}(\Omega)}^{2}=\int_{r=0}^{\frac{\alpha}{2}}(2 \pi r+2 \ell)\left(|q|^{2}+\left|q^{\prime}\right|^{2}\right) d r .
$$

As $q$ is an even function

$$
\|p\|_{H^{1}(\Omega)}^{2}=\int_{I_{\alpha}}\left(|q|^{2}+\left|q^{\prime}\right|^{2}\right) \omega(r) d r
$$

that is

$$
\|p\|_{H^{1}(\Omega)}=\|q\|_{H_{\omega}^{1}\left(I_{\alpha}\right)} .
$$

(ii) We can show equality (3.11) similarly fo $N=3$ : however

(1) we deal with triple integrals,

(2) the jacobian of $\Phi_{C}$ at $(t, r, \theta)$ is $|r(1-r \cos (\theta) \gamma(t))|$,

(3) the jacobian of $\Phi_{B^{0}}, \Phi_{B^{\ell}}$ at $(r, \theta, \phi)$ is $r^{2} \cos (\phi)$.

We set $D_{C}=[0, \ell] \times I_{\alpha} \times\left[-\frac{\pi}{2}, \frac{\pi}{2}\right]$ and $D_{B}=\left[0, \frac{\alpha}{2}\right] \times[0,2 \pi] \times\left[0, \frac{\pi}{2}\right]$; then

$$
\begin{aligned}
\|p\|_{H^{1}(\Omega)}^{2}= & \underbrace{\iiint_{(t, r, \theta) \in D_{c}}\left(|p|^{2}+|\nabla p|^{2}\right) \circ \Phi_{C}|r(1-r \cos (\theta) \gamma(t))|}_{I_{1}:=\text { Body }} \\
& +\underbrace{\iiint_{(r, \theta, \phi) \in D_{B}}\left(|p|^{2}+|\nabla p|^{2}\right) \circ \Phi_{B^{0}} r^{2} \cos (\phi)}_{I_{2}:=\text { end } B^{0}}, \\
& +\underbrace{\iiint_{(r, \theta, \phi) \in D_{B}}\left(|p|^{2}+|\nabla p|^{2}\right) \circ \Phi_{B^{\ell}} r^{2} \cos (\phi)}_{I_{3}:=\text { end } B^{\ell}},
\end{aligned}
$$


As previously we get with $(3.3)$

$$
\left.\forall r \in I_{\alpha}, \forall t \in 0, \ell\right] \quad|r(1-r \cos (\theta) \gamma(t))|=|r|(1-r \cos (\theta) \gamma(t)) .
$$

As $p$ is an even function with respect to $r$ we obtain

$$
\begin{aligned}
I_{1}= & \iiint_{(t, r, \theta) \in D_{C}^{+}}\left(|p|^{2}+|\nabla p|^{2}\right) \circ \Phi_{C}|r|(1-r \cos (\theta) \gamma(t)) d t d r d \theta \\
& +\iiint_{(t, r, \theta) \in D_{C}^{+}}\left(|p|^{2}+|\nabla p|^{2}\right) \circ \Phi_{C}|r|(1+r \cos (\theta) \gamma(t)) d t d r d \theta, \\
I_{1}= & 2 \iiint_{(t, r, \theta) \in D_{C}^{+}}\left(|p|^{2}+|\nabla p|^{2}\right) \circ \Phi_{C}|r| .
\end{aligned}
$$

where $D_{C}^{+}=D_{C} \cap\{r \geq 0\}$. With symmetry arguments we deduce

$$
I_{1}=\ell \pi \int_{I_{\alpha}}\left(|q|^{2}+\left|q^{\prime}\right|^{2}\right)|r| d r .
$$

We prove as in the $2 \mathrm{D}$-case that

$$
I_{2}=I_{3}=\pi \int_{I_{\alpha}}\left(|q|^{2}+\left|q^{\prime}\right|^{2}\right) r^{2} d r
$$

and

$$
\|p\|_{H^{1}(\Omega)}^{2}=\int_{I_{\alpha}}\left(\ell \pi|r|+2 \pi r^{2}\right)\left(|q|^{2}+\left|q^{\prime}\right|^{2}\right) d r .
$$

Equality (3.11) holds for $N=2,3$. This implies that $\Theta$ is an application from $\mathcal{F}_{\alpha}$ to $H_{\omega, 0}^{1}\left(I_{\alpha}\right)$. Moreover, with definition 3.2, $\Theta(p)$ is an even function that vanishes on $I_{\alpha}$ boundary, for every $p \in \mathcal{F}_{\alpha}$. More precisely, $\Theta\left(\mathcal{F}_{\alpha}\right) \subset \mathcal{G}_{\alpha}^{\omega}$. The bijectivity of $\Theta$ comes from definition 3.2 .

Remark 3.5. Let $\Sigma_{t}$ be the $t$ - slice of $A_{\alpha}$ :

$$
\Sigma_{t}= \begin{cases}\left\{\Phi_{C}(t, r): r \in I_{\alpha}\right\} & \text { if } N=2 \\ \left\{\Phi_{C}(t, r, \theta):(r, \theta) \in I_{\alpha} \times\right]-\frac{\pi}{2}, \frac{\pi}{2}[\} & \text { if } N=3\end{cases}
$$
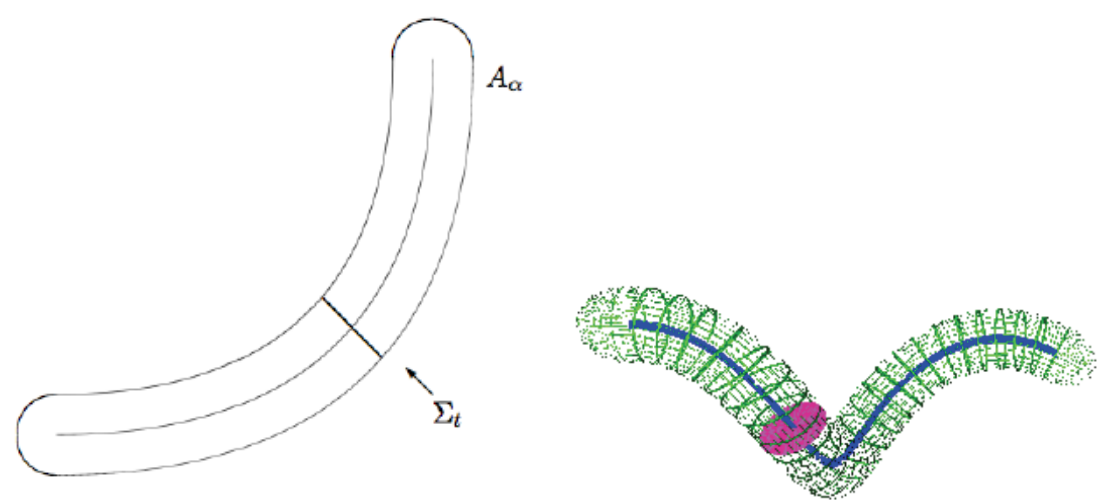

Figure 3.2. Slice $\Sigma_{t}$ for $N=2,3$

The bijectivity of $\Theta$ means that an element of $\mathcal{F}_{\alpha}$ is characterized by its image in the slice $\Sigma_{0}$ or any other slice $\Sigma_{t}$ of $A_{\alpha}$. This comes from the (strong) assumption we made on the geometry of the tube whose diameter $\alpha$ is constant. This assumption will be relaxed in the future to consider tubes with varying diameters. 
Now we can perform the change of variables $q=\Theta(p)$ that provides an equivalent 1D formulation of problem $\left(\mathcal{P}_{\varepsilon, \alpha}\right)$. We define $G_{\varepsilon}: \mathcal{G}_{\alpha}^{\omega} \rightarrow \mathbb{R}^{+}$as follows

$$
\forall q \in \mathcal{G}_{\alpha}^{\omega} \quad G_{\varepsilon}(q)=\mathcal{E}_{\varepsilon}\left(\Theta^{-1}(q)\right)
$$

Let us give an explicit expression of $G_{\varepsilon}$ :

Proposition 3.4. The function $G_{\varepsilon}: \mathcal{G}_{\alpha}^{\omega} \rightarrow \mathbb{R}^{+}$satisfies

$$
\forall q \in \mathcal{G}_{\alpha}^{\omega} \quad G_{\varepsilon}(q)=\int_{I_{\alpha}}\left[9 \varepsilon \beta\left|q^{\prime}\right|^{2}+\frac{1}{2}(1-q)^{2}+\frac{\left(q-q^{2}\right)^{2}}{\varepsilon} \beta\right] \omega(r) d r
$$

where $\omega$ has been defined in (3.10).

Proof. Let be $q \in \mathcal{G}_{\alpha}^{\omega}$ and $p=\Theta^{-1}(q) \in \mathcal{F}_{\alpha}$.

$$
\begin{aligned}
G_{\varepsilon}(q)=\mathcal{E}_{\varepsilon}(p) & =\frac{1}{2} \int_{\Omega}\left(p-\chi_{A_{\alpha}}\right)^{2} d r+\beta \int_{\Omega} 9 \varepsilon|\nabla p|^{2}+\frac{(p(p-1))^{2}}{\varepsilon} d r \\
& =\frac{1}{2} \int_{A_{\alpha}}(p-1)^{2} d r+\beta \int_{\Omega} 9 \varepsilon|\nabla p|^{2}+\frac{(p(p-1))^{2}}{\varepsilon} d r
\end{aligned}
$$

The computation is similar to the previous ones. We obtain (2D case)

$$
\begin{aligned}
G_{\varepsilon}(q)= & \ell \int_{-\frac{\alpha}{2}}^{\frac{\alpha}{2}} 9 \beta \varepsilon\left|q^{\prime}\right|^{2}+\frac{1}{2}(1-q)^{2}+\beta \frac{\left(q-q^{2}\right)^{2}}{\varepsilon} d r \\
& +\pi \int_{r=0}^{\frac{\alpha}{2}}\left(18 \beta \varepsilon\left|q^{\prime}\right|^{2}+(1-q)^{2}+2 \beta \frac{\left(q-q^{2}\right)^{2}}{\varepsilon}\right) r d r . \\
= & \int_{-\frac{\alpha}{2}}^{\frac{\alpha}{2}}\left(9 \beta \varepsilon\left|q^{\prime}\right|^{2}+\frac{1}{2}(1-q)^{2}+\beta \frac{\left(q-q^{2}\right)^{2}}{\varepsilon}\right)(\ell+\pi|r|) d r .
\end{aligned}
$$

The 3D computation is quite similar.

We call next reduced problem on $\mathcal{G}_{\alpha}^{\omega}$ the following

$$
\min _{p \in \mathcal{G}_{\alpha}^{\omega}} G_{\varepsilon}
$$

We just proved that we may reduce $\left(\mathcal{P}_{\varepsilon, \alpha}\right)$ to a $1 \mathrm{D}$ problem. More precisely:

Theorem 3.4. Assume that $\left(\mathcal{H}_{\Gamma}\right)$ and (3.3) are fulfilled.

The function $p$ is solution to $\left(\mathcal{P}_{\varepsilon, \alpha}\right)$ if and only if $\Theta(p)$ is solution to $\left(\mathcal{P}_{\varepsilon, \alpha}^{\omega}\right)$ where

- $\Theta$ is given by

$$
\begin{aligned}
\Theta: \mathcal{F}_{\alpha} & \rightarrow \mathcal{G}_{\alpha}^{\omega} \\
p & \mapsto\left\{\begin{array}{rll}
q: I_{\alpha} & \rightarrow \mathbb{R} \\
r & \mapsto p\left(\Phi_{C}(0, r)\right),
\end{array}\right.
\end{aligned}
$$

- $\mathcal{F}_{\alpha}$ is given by definition 3.2 and $\mathcal{G}_{\alpha}^{\omega}$ by definition 3.4

- $G_{\varepsilon}$ is given by (3.15) and $H_{\omega}^{1}\left(I_{\alpha}\right)$ by (3.3) with

- $2 D$ case $: \omega(r)=\ell+\pi|r|$ et

$$
\begin{aligned}
\left.\Phi_{C}:[0, \ell] \times\right]-\frac{\alpha}{2}, \frac{\alpha}{2}[ & \rightarrow C_{\alpha} \\
(t, r) & \mapsto F(t)+r N(t), \\
-3 D \text { case }: \omega(r)=\pi \ell r+2 \pi r^{2} \text { et } & \\
\Phi_{C} & :[0, \ell] \times]-\frac{\alpha}{2}, \frac{\alpha}{2}[\times]-\frac{\pi}{2}, \frac{\pi}{2}\left[\rightarrow C_{\alpha}\right. \\
(t, r, \theta)) & \mapsto F(t)+r \cos (\theta) N(t)+r \sin (\theta) B(t) .
\end{aligned}
$$


3.3. Solution properties. Theorem 3.4 is the key result of this paper: indeed we may now obtain quantitative and qualitative properties of the solution to $\left(\mathcal{P}_{\varepsilon, \alpha}\right)$ from the solution to $\left(\mathcal{P}_{\varepsilon, \alpha}^{\omega}\right)$.

With the symmetry properties of functions in $\mathcal{G}_{\alpha}^{\omega}$ it is easy to check that the restriction of the solution $\bar{q}$ of $\left(\mathcal{P}_{\varepsilon, \alpha}^{\omega}\right)$ ( with $\left.\beta \leq \varepsilon\right)$ to $\left(0, \frac{\alpha}{2}\right)$ is the unique solution to

$$
\min _{g \in \mathcal{G}_{\alpha}^{\omega,+}} G_{\varepsilon}^{+}(g)
$$

where

$$
\mathcal{G}_{\alpha}^{\omega,+}=\left\{q_{\left.\mid] 0, \frac{\alpha}{2}\right]}, \mid q \in \mathcal{G}_{\alpha}^{\omega}\right\}
$$

and

$G_{\varepsilon}^{+}(g)=\int_{0}^{\frac{\alpha}{2}}\left(9 \beta \varepsilon\left|g^{\prime}\right|^{2}+\frac{1}{2}(1-g)^{2}+\beta \frac{\left(g-g^{2}\right)^{2}}{\varepsilon}\right) \omega(r) d r=\int_{0}^{\frac{\alpha}{2}}\left(9 \beta \varepsilon\left|g^{\prime}\right|^{2}+F_{\beta, \varepsilon}(g)\right) \omega(r) d r$,

with the notations (3.7). We recall that $F_{\beta, \varepsilon}^{\prime}(t)=2 f_{\beta, \varepsilon}(t)$ where $f_{\beta, \varepsilon}$ is given by (3.8). We have seen that if $\beta \leq \varepsilon$ then $f_{\beta, \varepsilon}^{\prime} \geq 0$. Therefore, $F_{\beta, \varepsilon}^{\prime \prime}>0$ and $F_{\beta, \varepsilon}^{\prime}$ is increasing. As $F_{\beta, \varepsilon}^{\prime}(1)=0$, the function $F_{\beta, \varepsilon}^{\prime}$ is negative on $\left.]-\infty, 1\right]$ and nonnegative on $[1,+\infty[$. This proves that the function $F_{\beta, \varepsilon}$ is decreasing $\left.]-\infty, 1\right]$ and increasing on $[1,+\infty[$.

Theorem 3.5. Assume $\beta \leq \varepsilon$. Let $\bar{p}$ be the unique solution to $\left(\mathcal{P}_{\varepsilon, \alpha}\right)$ and $\bar{q}=\Theta(\bar{p})$. Then $\bar{q}$ (and $\bar{p})$ takes its values in $[0,1]$. In particular $\bar{q} \in L^{\infty}\left(I_{\alpha}\right)$.

Proof. It is sufficient to prove that

$$
\left.\forall r \in] 0, \frac{\alpha}{2}\right] \quad 0 \leq \bar{q}(r) \leq 1 .
$$

Lemmas 3.2 and 3.3 ensure that $\bar{q}$ is continuous on $\left.] 0, \frac{\alpha}{2}\right]$ in the $3 \mathrm{D}$ case and continuous on $\left[0, \frac{\alpha}{2}\right]$ in the $2 \mathrm{D}$ case. So if $N=2$, we get $0 \leq \bar{q}(0) \leq 1$ by continuity.

- Let us prove first that

$$
\left.\forall r \in] 0, \frac{\alpha}{2}\right], \bar{q}(r) \leq 1 .
$$

Define $\varphi=\min (\bar{q}, 1)$ on $\left.] 0, \frac{\alpha}{2}\right]$ so that $\varphi \in \mathcal{G}_{\alpha}^{\omega,+}$. Then

$$
\int_{0}^{\frac{\alpha}{2}} 9 \beta \varepsilon\left|\varphi^{\prime}\right|^{2} \omega(r) d r \leq \int_{0}^{\frac{\alpha}{2}} 9 \beta \varepsilon\left|\bar{q}^{\prime}\right|^{2} \omega(r) d r .
$$

From the other hand, $F_{\beta, \varepsilon}$ is increasing on $[1,+\infty[$ so that

$$
\begin{array}{ll}
F_{\beta, \varepsilon}(\varphi(r))=F_{\beta, \varepsilon}(\bar{q}(r)) & \text { if } \bar{q}(r)) \leq 1, \\
F_{\beta, \varepsilon}(\varphi(r)) \leq F_{\beta, \varepsilon}(\bar{q}(r)) & \text { if } \bar{q}(r)) \geq 1=\varphi(r),
\end{array}
$$

As $\omega \geq 0$ it comes

$$
G_{\varepsilon}^{+}(\varphi) \leq G_{\varepsilon}^{+}(\bar{q})
$$

and with the uniqueness of the solution, this yields : $\varphi=\bar{q}$. Therefore $\bar{q} \leq 1$.

- We prove similarly that

$$
\left.\forall r \in] 0, \frac{\alpha}{2}\right], \bar{q}(r) \geq 0 .
$$

Set $\varphi=\max (\bar{q}, 0)$ on $\left.] 0, \frac{\alpha}{2}\right]$ so that $\varphi \in \mathcal{G}_{\alpha}^{\omega,+}$ and

$$
\int_{0}^{\frac{\alpha}{2}} 9 \beta \varepsilon\left|\varphi^{\prime}\right|^{2} \omega(r) d r \leq \int_{0}^{\frac{\alpha}{2}} 9 \beta \varepsilon\left|\bar{q}^{\prime}\right|^{2} \omega(r) d r .
$$

Furthermore

$$
\begin{array}{ll}
F_{\beta, \varepsilon}(\varphi(r))=F_{\beta, \varepsilon}(\bar{q}(r)) & \text { if } \bar{q}(r)) \geq 0 \\
1=F_{\beta, \varepsilon}(0) \leq F_{\beta, \varepsilon}(\bar{q}(r)) & \text { if } \bar{q}(r)) \leq 0
\end{array}
$$


since $F_{\beta, \varepsilon}$ is decreasing on $\left.]-\infty, 0\right]$. As $\omega \geq 0$ we get

$$
G_{\varepsilon}^{+}(\varphi) \leq G_{\varepsilon}^{+}(\bar{q})+\int_{\bar{q} \leq 0}(1-F(\bar{q})) \omega(r) d r \leq G_{\varepsilon}^{+}(\bar{q}) .
$$

As before $\varphi=\bar{q}$ and $\bar{q} \geq 0$.

Now, we make the optimality condition precise : let $\bar{p}$ be the unique solution to $\left(\mathcal{P}_{\varepsilon, \alpha}\right)$ and $\bar{q}=\Theta(\bar{p})$. Then

$$
\forall \psi \in \mathcal{G}_{\alpha}^{\omega} \quad \int_{I_{\alpha}}\left(9 \beta \varepsilon \bar{q}^{\prime} \psi^{\prime}+f_{\beta, \varepsilon}(q) \psi\right) \omega(r) d r=0 .
$$

Let us denote

$$
\mathcal{H}_{\omega}^{1}\left(0, \frac{\alpha}{2}\right):=\left\{\varphi \in H_{\omega}^{1}\left(0, \frac{\alpha}{2}\right), \varphi\left(\frac{\alpha}{2}\right)=0\right\},
$$

where $\omega$ is defined with (3.10). It is a linear subspace of $\mathcal{C}^{0}\left(\left[0, \frac{\alpha}{2}\right]\right)$ for $N=2$ and $\left.\left.\mathcal{C}^{0}(] 0, \frac{\alpha}{2}\right]\right)$ for $N=3$. For every function $\varphi \in \mathcal{H}_{\omega}^{1}\left(0, \frac{\alpha}{2}\right)$ we set

$$
\psi(x)= \begin{cases}\varphi(x) & \text { if } x>0 \\ \varphi(-x) & \text { if } x<0\end{cases}
$$

The function $\psi$ belongs to $\mathcal{G}_{\alpha}^{\omega}$ and with (3.17)

Finally

$$
\begin{gathered}
\int_{-\frac{\alpha}{2}}^{\frac{\alpha}{2}}\left(9 \beta \varepsilon \bar{q}^{\prime} \psi^{\prime}+f_{\beta, \varepsilon}(\bar{q}) \psi\right) \omega(r) d r=2 \int_{0}^{\frac{\alpha}{2}}\left(9 \beta \varepsilon \bar{q}^{\prime} \psi^{\prime}+f_{\beta, \varepsilon}(\bar{q}) \psi\right) \omega(r) d r \\
=2 \int_{0}^{\frac{\alpha}{2}}\left(9 \beta \varepsilon \bar{q}^{\prime} \varphi^{\prime}+f_{\beta, \varepsilon}(\bar{q}) \varphi\right) \omega(r) d r=0 .
\end{gathered}
$$

$$
\forall \varphi \in \mathcal{H}_{\omega}^{1}\left(0, \frac{\alpha}{2}\right) \quad \int_{0}^{\frac{\alpha}{2}}\left(9 \beta \varepsilon \bar{q}^{\prime}(r) \varphi^{\prime}(r)+f_{\beta, \varepsilon}(\bar{q}(r)) \varphi(r)\right) \omega(r) d r=0 .
$$

Choose $\varphi \in \mathcal{D}\left(0, \frac{\alpha}{2}\right)$ and integrate by parts gives

$$
-9 \beta \varepsilon\left(\omega \bar{q}^{\prime}\right)^{\prime}+\omega f_{\beta, \varepsilon}(\bar{q})=0 \text { in }\left(0, \frac{\alpha}{2}\right),
$$

in the sense of distributions. In addition $\bar{q}\left(\frac{\alpha}{2}\right)=0$.

Now, choose $\varphi \in \mathcal{C}^{1}\left(0, \frac{\alpha}{2}\right)$ such that $\varphi\left(\frac{\alpha}{2}\right)=0$ and $\varphi(0) \neq 0$. An integration by parts gives

$$
\int_{0}^{\frac{\alpha}{2}} \bar{q}^{\prime} \varphi^{\prime} \omega(r) d r=-\int_{0}^{\frac{\alpha}{2}}\left(\omega \bar{q}^{\prime}\right)^{\prime} \varphi d r-\bar{q}^{\prime}(0) \omega(0) \varphi(0),
$$

and with (3.18) we obtain $\bar{q}^{\prime}(0) \omega(0) \varphi(0)=0$, that is $\bar{q}^{\prime}(0) \omega(0)=0$. Consequently, if $N=2$ $(\omega(0)=\ell \neq 0)$ we get $q^{\prime}(0)=0$ and we may describe the $2 \mathrm{D}$ solution.

\section{4. $2 \mathrm{D}$ case.}

Theorem 3.6 (Euler equation ). Assume $\beta \leq \varepsilon$. Let $\bar{p}$ be the unique solution to $\left(\mathcal{P}_{\varepsilon, \alpha}\right)$ and $\bar{q}=\Theta(\bar{p})$. Then $\bar{q} \in \mathcal{G}_{\alpha}^{\omega}$ is solution to the boundary problem

$$
\left\{\begin{array}{l}
-9 \beta \varepsilon\left(\omega \bar{q}^{\prime}\right)^{\prime}+\omega f_{\beta, \varepsilon}(\bar{q})=0 \text { in }\left(0, \frac{\alpha}{2}\right) \\
\bar{q}\left(\frac{\alpha}{2}\right)=0, \bar{q}^{\prime}(0)=0
\end{array}\right.
$$

In that case $\bar{q} \in \mathcal{C}^{2}\left(I_{\alpha}\right)$ and is the (strong) solution to

$$
\left\{\begin{array}{l}
-9 \beta \varepsilon\left(\omega \bar{q}^{\prime}\right)^{\prime}+\omega f_{\beta, \varepsilon}(\bar{q})=0 \text { in } I_{\alpha}, \\
\bar{q}\left(-\frac{\alpha}{2}\right)=\bar{q}\left(\frac{\alpha}{2}\right)=0 .
\end{array}\right.
$$

Moreover $\bar{q}^{\prime}(0)=0$. 
Proof. We have seen that $\bar{q}$ is the solution of (3.19) in the sense of distributions. As $\bar{q} \in H^{1}\left(I_{\alpha}\right)$ is continuous on $I_{\alpha}$ the equation above can be extended by parity and gives the system (3.20). Since $\bar{q} \in H^{1}\left(I_{\alpha}\right) \subset L^{\infty}\left(I_{\alpha}\right)$, the function

$$
\left\{\begin{aligned}
I_{\alpha} & \rightarrow \mathbb{R} \\
r & \mapsto \omega(r) f_{\beta, \varepsilon}(\bar{q})(r),
\end{aligned}\right.
$$

belongs to $L^{2}\left(I_{\alpha}\right)$. From (3.19) and (3.21), it can be deduced that $\left(\varepsilon \beta \omega \bar{q}^{\prime}\right)^{\prime} \in L^{2}\left(I_{\alpha}\right)$ and $\omega \bar{q}^{\prime} \in H^{1}\left(I_{\alpha}\right)$. As, $H^{1}\left(I_{\alpha}\right) \subset \mathcal{C}^{0}\left(I_{\alpha}\right)$, then $\omega \bar{q}^{\prime}$ is continuous. Dividing by $\omega$ (which does not vanish), we deduce that $\bar{q}^{\prime}$ is continuous on $I_{\alpha}$. In other words, $\bar{q} \in \mathcal{C}^{1}\left(I_{\alpha}\right)$.

We use the same reasoning to prove with $q \in \mathcal{C}^{1}\left(I_{\alpha}\right)$ and relation (3.17) that $\left(\varepsilon \beta \omega \bar{q}^{\prime}\right)^{\prime}$ is $\mathcal{C}^{1}$. On the other hand,

$$
\left(\omega \bar{q}^{\prime}\right)^{\prime}=\pi H \bar{q}^{\prime}+\omega \bar{q}^{\prime \prime}
$$

in the distributional sense, where $H$ is the Heaviside function $\left(H \equiv-1\right.$ on $\mathbb{R}^{-}$and $H \equiv 1$ on $\mathbb{R}^{+}$). We noticed that $\bar{q}^{\prime}(0)=0$ : therefore $\pi H \bar{q}^{\prime}$ is continuous. This implies that $\omega \bar{q}^{\prime \prime}$ is continuous as well. Dividing once again by $\omega$, we claim that $\bar{q}^{\prime \prime}$ is a continuous function. Therefore $\bar{q} \in C^{2}\left(I_{\alpha}\right)$ is a strong solution of (3.17).

We now precise the solution shape. Indeed, we want it to be as close as possible to the indicator function of $I_{\alpha}$. We are going to prove that the solution shape is as in Figure 3.3. Therefore we have to estimate $\bar{q}(0)$ and $\bar{q}^{\prime}\left(\frac{\alpha}{2}\right)$ to tune parameters $\beta$ and $\varepsilon$ so that $\bar{q}(0)$ is as close as possible to 1 and $\left|\bar{q}^{\prime}\left(\frac{\alpha}{2}\right)\right|$ as large as possible.

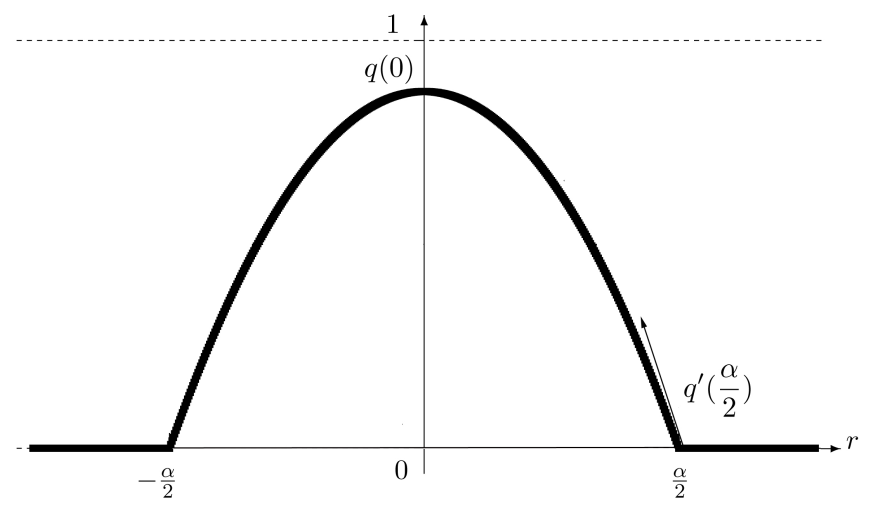

FiguRE 3.3. Solution for $N=2$

Theorem 3.7. Assume $N=2$ and $\beta \leq \varepsilon$. Let $\bar{p}$ be the unique solution to $\left(\mathcal{P}_{\varepsilon, \alpha}\right)$ and $\bar{q}=\Theta(\bar{p})$. Then

(1) $\bar{q}$ is an even function that is decreasing on $\left[0, \frac{\alpha}{2}\right]$,

$$
\bar{q}(0)-\frac{1}{36 \beta \varepsilon} t^{2}+\circ\left(t^{2}\right) \leq \bar{q}(t) \leq \bar{q}(0)
$$

$$
-f_{\beta, \varepsilon}(\bar{q}(0)) \frac{\alpha^{2}}{144 \beta \varepsilon} \leq \bar{q}(0) \leq \frac{\alpha^{2}}{144 \beta \varepsilon}
$$

$$
-\frac{\alpha}{36 \beta \varepsilon} \leq \bar{q}^{\prime}\left(\frac{\alpha}{2}\right) \leq f_{\beta, \varepsilon}(\bar{q}(0)) \frac{\alpha}{36 \beta \varepsilon}
$$


(5)

$$
q^{\prime \prime}\left(\frac{\alpha}{2}\right) \leq 0
$$

where $f_{\beta, \varepsilon}$ is given by (3.8).

Proof. Let $\bar{p}$ be the unique solution to $\left(\mathcal{P}_{\varepsilon, \alpha}\right)$ and $\bar{q}=\Theta(\bar{p})$. We denote $q=\bar{q}_{\mid\left[0, \frac{\alpha}{2}\right]}$ the solution to problem (3.16).

(1) As $\bar{q}$ is even, it is sufficient to prove that $q$ is decreasing on $\left[0, \frac{\alpha}{2}\right]$. We have proved that $q$ is the strong solution of

$$
9 \beta \varepsilon\left(\omega q^{\prime}\right)^{\prime}=\omega f_{\beta, \varepsilon}(q) \text { in }\left[0, \frac{\alpha}{2}\right] .
$$

As $q \leq 1$ and $f_{\beta, \varepsilon}$ is increasing with $f_{\beta, \varepsilon}(1)=0$ we get

$$
\forall r \in\left[0, \frac{\alpha}{2}\right] \quad \omega(r) f_{\beta, \varepsilon}(q)(r) \leq 0 .
$$

Therefore $\left(\omega q^{\prime}\right)^{\prime}$ is a continuous, negative function on $\left[0, \frac{\alpha}{2}\right]$ and $\omega q^{\prime}$ is decreasing. In particular

$$
\forall r \in\left[0, \frac{\alpha}{2}\right] \quad \omega(r) q^{\prime}(r) \leq \omega(0) q^{\prime}(0)=0 .
$$

Thus $q^{\prime} \leq 0$ and $q$ is decreasing.

(2) Let us perform a local study at $t=0$. With equation (3.19) we have

$$
\forall t \in\left[0, \frac{\alpha}{2}\right] \quad 9 \beta \varepsilon \int_{0}^{t}\left(\omega(s) \bar{q}^{\prime}(s)\right)^{\prime} d s=\int_{0}^{t} \omega(s) f_{\beta, \varepsilon}(\bar{q}(s)) d s .
$$

Let us estimate

$$
\int_{0}^{t} \omega(s) f_{\beta, \varepsilon}(\bar{q}(s)) d s .
$$

As $\bar{q}$ is decreasing on $[0, t]$, takes its values in $[0,1]$ and $f_{\beta, \varepsilon}$ is an increasing, negative function, we have: $\forall 0 \leq s \leq t \leq \frac{\alpha}{2}$

$$
-\frac{1}{2}=f_{\beta, \varepsilon}(0) \leq f_{\beta, \varepsilon}(\bar{q}(t)) \leq f_{\beta, \varepsilon}(\bar{q}(s)) \leq f_{\beta, \varepsilon}(\bar{q}(0)) \leq 0
$$

and (with $\omega \geq 0$ )

$$
f_{\beta, \varepsilon}(\bar{q}(t)) \int_{0}^{t} \omega(s) d s \leq \int_{0}^{t} \omega(s) f_{\beta, \varepsilon}(\bar{q}(s)) d s \leq f_{\beta, \varepsilon}(\bar{q}(0)) \int_{0}^{t} \omega(s) d s \leq 0,
$$

that is

$$
f_{\beta, \varepsilon}(\bar{q}(t)) \frac{(\ell+\pi t)^{2}-\ell^{2}}{2 \pi} \leq 9 \beta \varepsilon \omega(t) \bar{q}^{\prime}(t) \leq f_{\beta, \varepsilon}(\bar{q}(0)) \frac{(\ell+\pi t)^{2}-\ell^{2}}{2 \pi},
$$

since $\bar{q}^{\prime}(0)=0$. Thus we obtain

$$
f_{\beta, \varepsilon}(\bar{q}(t)) \frac{(2 \ell+\pi t) t}{2} \leq 9 \beta \varepsilon(\ell+\pi t) \bar{q}^{\prime}(t) \leq f_{\beta, \varepsilon}(\bar{q}(0)) \frac{(2 \ell+\pi t) t}{2},
$$

that is finally

$$
\forall t \in\left[0, \frac{\alpha}{2}\right] \quad \lambda(t) f_{\beta, \varepsilon}(\bar{q}(t)) \frac{t}{18 \beta \varepsilon} \leq \bar{q}^{\prime}(t) \leq \lambda(t) f_{\beta, \varepsilon}(\bar{q}(0)) \frac{t}{18 \beta \varepsilon},
$$

where we have set

$$
\lambda(t)=\frac{(2 \ell+\pi t)}{\ell+\pi t}=1+\frac{\ell}{\ell+\pi t} .
$$


By continuity, we get

$$
\bar{q}^{\prime \prime}(0)=\lim _{t \rightarrow 0^{+}} \frac{\bar{q}(t)}{t}=\frac{f_{\beta, \varepsilon}(\bar{q}(0))}{9 \beta \varepsilon},
$$

and a local expansion of $\bar{q}$ at 0 as well:

$$
\bar{q}(t)=\bar{q}(0)+\frac{f_{\beta, \varepsilon}(\bar{q}(0))}{18 \beta \varepsilon} t^{2}+\circ\left(t^{2}\right),
$$

since $q^{\prime}(0)=0$. As $0 \geq f_{\beta, \varepsilon}(\bar{q}(0)) \geq-\frac{1}{2}$ we obtain inequality (3.22).

(3) Equations (3.25), (3.26) and $1 \leq \lambda(t) \leq 2$ give

$$
\forall t \in\left[0, \frac{\alpha}{2}\right] \quad-1 \leq \lambda(t) f_{\beta, \varepsilon}(\bar{q}(t)) \text { and } \lambda(t) f_{\beta, \varepsilon}(\bar{q}(0)) \leq f_{\beta, \varepsilon}(\bar{q}(0)) .
$$

This yields

$$
\forall t \in\left[0, \frac{\alpha}{2}\right] \quad-\frac{t}{18 \beta \varepsilon} \leq \bar{q}^{\prime}(t) \leq f_{\beta, \varepsilon}(\bar{q}(0)) \frac{t}{18 \beta \varepsilon} .
$$

Performing an integration between 0 and $\frac{\alpha}{2}$ gives

$$
-\frac{\alpha^{2}}{8 * 18 \beta \varepsilon} \leq \bar{q}\left(\frac{\alpha}{2}\right)-\bar{q}(0) \leq f_{\beta, \varepsilon}(\bar{q}(0)) \frac{\alpha^{2}}{8 * 18 \beta \varepsilon},
$$

and with $\bar{q}\left(\frac{\alpha}{2}\right)=0$ :

$$
-f_{\beta, \varepsilon}(\bar{q}(0)) \frac{\alpha^{2}}{144 \beta \varepsilon} \leq \bar{q}(0) \leq \frac{\alpha^{2}}{144 \beta \varepsilon} .
$$

(4) $q^{\prime}\left(\frac{\alpha}{2}\right)$ estimate. Equation (3.26) with $t=\frac{\alpha}{2}$ provides

$$
2 f_{\beta, \varepsilon}\left(\bar{q}\left(\frac{\alpha}{2}\right)\right) \frac{\alpha}{2 * 18 \beta \varepsilon} \leq \bar{q}^{\prime}\left(\frac{\alpha}{2}\right) \leq f_{\beta, \varepsilon}(\bar{q}(0)) \frac{\alpha}{2 * 18 \beta \varepsilon}
$$

since $1 \leq \lambda(t) \leq 2$. As $f_{\beta, \varepsilon}\left(\bar{q}\left(\frac{\alpha}{2}\right)\right)=f_{\beta, \varepsilon}(0)=-\frac{1}{2}$ we have proved relation (3.24).

(5) We finally prove that $q^{\prime \prime}\left(\frac{\alpha}{2}\right) \leq 0$. The differential equation writes

$$
\forall t \in] 0, \frac{\alpha}{2}\left[\quad-9 \beta \varepsilon \pi \bar{q}^{\prime}(t)-9 \beta \varepsilon(\ell+\pi t) \vec{q}^{\prime \prime}(t)+(\ell+\pi t) f_{\beta, \varepsilon}(\bar{q}(t))=0 .\right.
$$

Passing to the limit as $t \rightarrow \frac{\alpha}{2}$, we obtain

$$
\vec{q}^{\prime}\left(\frac{\alpha}{2}\right)=-\frac{\pi}{\ell+\pi \frac{\alpha}{2}} \bar{q}^{\prime}\left(\frac{\alpha}{2}\right)-\frac{1}{18 \beta \varepsilon}\left(\ell+\pi \frac{\alpha}{2}\right) .
$$

Equation (3.24) yields

$$
0 \leq-\bar{q}^{\prime}\left(\frac{\alpha}{2}\right) \leq \frac{\alpha}{36 \beta \varepsilon}
$$

So

$$
\vec{q}^{\prime \prime}\left(\frac{\alpha}{2}\right) \leq \frac{\alpha \pi}{36 \beta \varepsilon} \frac{1}{\left(\ell+\pi \frac{\alpha}{2}\right)}-\frac{1}{18 \beta \varepsilon} \leq \frac{-2 \ell}{36 \beta \varepsilon\left(\ell+\pi \frac{\alpha}{2}\right)} \leq 0
$$

The previous theorem allows to tune parameters with respect to the diameter $\alpha$, so that the solution is as close as possible of the indicator of $I_{\alpha}$. 
Corollary 3.1. Parameters $\beta$ and $\varepsilon$ should be chosen such that

$$
\beta \leq \varepsilon, \frac{1}{36}<<\beta \varepsilon<<\alpha, \beta \varepsilon<\frac{\alpha^{2}}{144} .
$$

If we introduce $\gamma$ as the fitting data term parameter (so that $\beta$ becomes $\frac{\beta}{\gamma}$ ) then

$$
\beta \leq \gamma \varepsilon, \frac{\gamma}{36}<<\beta \varepsilon<<\gamma \alpha, \beta \varepsilon<\gamma \frac{\alpha^{2}}{144} .
$$

If we choose $\gamma=\frac{1}{\alpha}$ for example, one gets

$$
\beta \leq \frac{\varepsilon}{\alpha}, \frac{1}{36 \alpha}<<\beta \varepsilon<<1, \beta \varepsilon<\frac{\alpha}{144} .
$$

Proof. We have to set $\beta \leq \varepsilon$ to get uniqueness. If we want the solution to be very flat at 0 it is sufficient to set

$$
\beta \varepsilon>>\frac{1}{36} .
$$

with (3.22). We would like $\bar{q}(0)$ to be close to 1 . Passing to the limit as $t \rightarrow 1$ in relation (3.22) gives a necessary condition

$$
\alpha^{2} \geq 144 \beta \varepsilon
$$

The right inequality in (3.24) is not useful : indeed, ideally $\bar{q}(0)=1$ and $f_{\beta, \varepsilon}(\bar{q}(0))=0$. However $\left|\bar{q}^{\prime}\left(\frac{\alpha}{2}\right)\right|$ can be large only if the left hand side is. ne grand si de gauche . A necessary condition is

$$
\beta \varepsilon<<\alpha .
$$

3.5. 3D case. Unisng the same techniques as in the $2 \mathrm{D}$ case, one can prove that the solution $\bar{q}$ is $\mathcal{C}^{2}$ on $\left.] 0, \frac{\alpha}{2}\right]$ and is a strong solution to

$$
\left\{\begin{array}{l}
-9 \beta \varepsilon\left(\omega \bar{q}^{\prime}\right)^{\prime}+\omega f_{\beta, \varepsilon}(\bar{q})=0 \text { in }\left[\eta, \frac{\alpha}{2}\right] \\
\bar{q}(\eta) \text { given }, \bar{q}\left(\frac{\alpha}{2}\right)=0 .
\end{array}\right.
$$

for every $\left.\eta \in] 0, \frac{\alpha}{2}\right]$. Nevertheless, one cannot conclude that $\bar{q}$ is $\mathcal{C}^{1}$ on $\left[0, \frac{\alpha}{2}\right]$ because $\omega(0)=0$ and the solution may be singular at 0 . However, we may use the following strategy

- The $3 \mathrm{D}$ problem is equivalent to a $2 \mathrm{D}$ one with the same projection technique. A weight function $\omega_{2}$ appear with $\omega_{2}(0) \neq 0$.

- Then we pass from $2 \mathrm{D}$ to $1 \mathrm{D}$ by noticing that the $2 \mathrm{D}$ problem owns symmetry properties once again.

We will not detail this strategy and rather present a slightly modified model that allows to give regularity results in the $3 \mathrm{D}$ case.

\section{A MODIFIED 3D MODEL}

The possible singularity of teh soltuion at 0 comes from the fact that $\omega(0)=0$. This is due to the ends of the tube contribution to $\omega$. Therefore, we consider a modified tube model where ends are excluded. 
4.1. Modeling the tube. As in section 3., assumptions $\left(\mathcal{H}_{\Gamma}\right)$ are needed to define a Frenet-Serret frame. Thickness around $\Gamma$ is defined now: it is said that the section of the tube along $\Gamma$ is less than $\alpha$ (diameter of the tube) if the points are at a distance less than $\frac{\alpha}{2}$ of $\Gamma$. Once again it is required that the radius of curvature is not too small and we assume (3.3). We have a result similar to the one of section 3 .

Proposition 4.1. Assume $\left(\mathcal{H}_{\Gamma}\right)$ and (3.3) are satisfied. Then the following application $\Phi$ is a local diffeomorphism:

$$
\begin{array}{rll}
\Phi & : \quad] 0, \ell[\times] 0, \frac{\alpha}{2}[\times]-\pi, \pi[\rightarrow \Omega \\
(t, r, \theta) & \mapsto \quad F(t)+r \cos (\theta) N(t)+r \sin (\theta) B(t) .
\end{array}
$$

The Jacobian of $\Phi$ is

$$
J \Phi(t, r, \theta)=r(1-r \cos (\theta) \gamma(t)) .
$$

The proof is similar to the one of proposition 3.2.

Definition 4.1. Let be $\Phi$ as in proposition 4.1 and $T_{\alpha}$ the range of $\Phi$. We say $T_{\alpha}$ is a tube if $\Phi$ is a global diffeomorphism from $] 0, \ell[\times] 0, \frac{\alpha}{2}[\times]-\pi, \pi\left[\right.$ onto $T_{\alpha}$.

The (new) space $\mathcal{F}_{\alpha}$ is the space of $H_{0}^{1}(\Omega)$ functions $p$ with support in $T_{\alpha}$ such that

$$
p(\Phi(t, r, \theta))=p(\Phi(t, r, \tilde{\theta}) \quad t \in] 0, \ell[\text {, a.e. } r \in] 0, \frac{\alpha}{2}[\text {, a.e. }(\theta, \tilde{\theta}) \in]-\pi, \pi\left[^{2}\right. \text {. }
$$

\section{Commenter}

We now consider a minimization problem to detect such a tube $T_{\alpha}$.

$$
\min _{p \in \mathcal{F}_{\alpha}} \mathcal{E}_{\varepsilon}(p)
$$

where

$$
\mathcal{E}_{\varepsilon}(p)=\frac{1}{2} \int_{T_{\alpha}}(p-1)^{2} d x+\beta \int_{T_{\alpha}}\left(9 \varepsilon|\nabla p|^{2}+\frac{p^{2}(1-p)^{2}}{\varepsilon}\right) d x .
$$

The following result can be proved as in section 3.1.4

Theorem 4.1. Problem $\left(\mathcal{Q}_{\varepsilon, \alpha}\right)$ has at least a solution. This solution is unique if $\beta \leq \varepsilon$. Moreover, any solution $\bar{p}$ of $\left(\mathcal{P}_{\varepsilon, \alpha}\right)$ satisfies

$$
\forall \varphi \in \mathcal{F}_{\alpha} \quad \int_{T_{\alpha}}\left(9 \beta \varepsilon \nabla \bar{p} \nabla \varphi+f_{\beta, \varepsilon}(p) \varphi\right) d x=0
$$

where $f_{\beta, \varepsilon}$ is defined with (3.8).

4.2. 3D tube rectification. The purpose of this section is to show that with the symmetry assumptions (4.1) the minimization problem is equivalent to minimizing the functional on the set of zero curvature tubes $T_{\alpha}^{\star}$, i.e. the ones for which $\Gamma$ is a segment. 

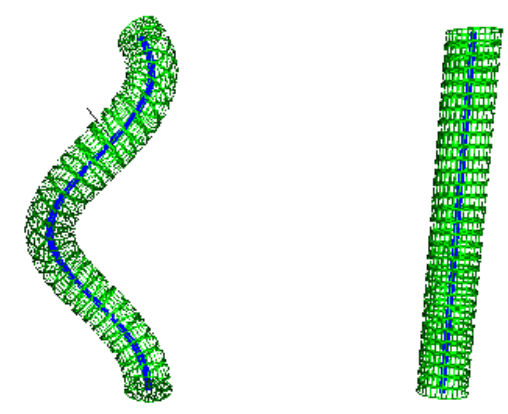

FiguRE 4.1. Tube rectification

Definition 4.2. To consider the rectified problem we set:

- $\Gamma^{\star}$ the $\mathbb{R}^{3}$-segment given by

$$
\begin{aligned}
F^{\star}:[0, \ell] & \rightarrow \mathbb{R}^{3}, \\
t & \rightarrow(t, 0,0) .
\end{aligned}
$$

- $T_{\alpha}^{\star}$ the $\alpha$ diameter tube associated to $\Gamma^{\star}$ :

$$
T_{\alpha}^{\star}=\left\{x \in \mathbb{R}^{3} \mid x=(t, r \cos \theta, r \sin \theta), t \in[0, \ell], r \in\left[0, \frac{\alpha}{2}\right], \theta \in[-\pi, \pi]\right\} .
$$

- $\mathcal{F}_{\alpha}^{\star} \subset H_{0}^{1}\left(T_{\alpha}^{\star}\right)$ the space of functions $q$ with support in $T_{\alpha}^{\star}$ such that for almost every $(t, r) \in[0, \ell] \times\left[0, \frac{\alpha}{2}\right]$ and $\left(\theta_{1}, \theta_{2}\right) \in \mathbb{R}^{2}$ :

$$
q\left(t, r \cos \theta_{1}, r \sin \theta_{1}\right)=q\left(t, r \cos \theta_{2}, r \sin \theta_{2}\right),
$$

- $\omega: T_{\alpha}^{\star} \rightarrow \mathbb{R}$ such that

$$
\omega(t, r \cos \theta, r \sin \theta)=\frac{1}{1-r^{2}(\cos \theta)^{2} \gamma(t)^{2}},
$$

- $\mathbb{A}_{\omega}: T_{\alpha}^{\star} \rightarrow M_{3,3}(\mathbb{R})$ such that

$$
\mathbb{A}_{\omega}(t, r \cos \theta, r \sin \theta)=\left(\begin{array}{ccc}
\sqrt{\omega(t, r \cos \theta, r \sin \theta)} & 0 & 0 \\
0 & 1 & 0 \\
0 & 0 & 1
\end{array}\right),
$$

- $\mathcal{E}_{\varepsilon}^{\star}$ the energy functional defined on $\mathcal{F}_{\alpha}^{\star}$ by

$$
\mathcal{E}_{\varepsilon}^{\star}(q)=\frac{1}{2} \int_{T_{\alpha}^{\star}}(q-1)^{2} d x+\beta \int_{T_{\alpha}^{\star}}\left(9 \varepsilon|A \nabla q|^{2}+\frac{(q(q-1))^{2}}{\varepsilon}\right) d x,
$$

- $H_{\omega}^{1}\left(T_{\alpha}^{\star}\right)$ the space of measurable functions such that

$$
\|p\|_{H_{\omega}^{1}}^{2}=\int_{T_{\alpha}^{\star}}\left(\left|\mathbb{A}_{\omega} \nabla p\right|^{2}+p^{2}\right) d x<+\infty,
$$

Let $\left(\mathcal{Q}_{\varepsilon, \alpha}^{\star}\right)$ be the minimization problem

$$
\min _{q \in \mathcal{F}_{\alpha}^{\star}} E_{\varepsilon}^{\star}(q) .
$$

With assumptions of proposition 4.1 and (3.3) we get :

$$
0 \leq r \leq \frac{\alpha}{2}, t \in[0, \ell] \Rightarrow 0 \leq r \gamma(t)<\frac{1}{2}
$$


Therefore the application $\omega$ makes sense and takes its values in $\left[1, \frac{4}{3}\right]$. This proves that

$$
H^{1}\left(T_{\alpha}^{\star}\right)=H_{\omega}^{1}\left(T_{\alpha}^{\star}\right)
$$

Now we may define $\Theta$ as

$$
\begin{aligned}
\Theta: T_{\alpha} & \rightarrow T_{\alpha}^{\star} \\
x & \mapsto(t, r \cos \theta, r \sin \theta),
\end{aligned}
$$

where $x=\Phi(t, r, \theta)$. As $\Phi$ is a diffeomorphism (proposition 4.1) and the polar coordinates parametrization as well, then $\Theta$ is also a diffeomorphism .

Proposition 4.2. The following application is an isomorphism :

$$
\begin{aligned}
\Psi: \mathcal{F}_{\alpha} & \rightarrow \mathcal{F}_{\alpha}^{\star} \\
p & \mapsto p \circ \Theta .
\end{aligned}
$$

Moreover $\|p\|_{H^{1}(\Omega)}=\|\Psi(p)\|_{H_{\omega}^{1}\left(T_{\alpha}^{\star}\right)}$ and $\mathcal{E}_{\varepsilon}(p)=\mathcal{E}_{\varepsilon}^{\star}(\Psi(p))$.

Proof. Let be $p \in \mathcal{F}_{\alpha}$. As the support of $p$ is included in $T_{\alpha}$, we have

$$
\|p\|_{H^{1}(\Omega)}^{2} d x=\iiint_{\Omega}\left(|p|^{2}+|\nabla p|^{2}\right) d x=\iiint_{T_{\alpha}}\left(|p|^{2}+|\nabla p|^{2}\right) d x
$$

We know that $\Phi$ is a parametrization of $T_{\alpha}$. Denoting $\left.D=\right] 0, \ell[\times] 0, \frac{\alpha}{2}[\times]-\pi, \pi[$, and $\mathbf{x}=(t, r, \theta)$

$$
\|p\|_{H^{1}(\Omega)}^{2}=\iiint_{\mathbf{x} \in D}\left(|p \circ \Phi|^{2}+|\nabla p \circ \Phi|^{2}\right)|r(1-r \cos (\theta) \gamma(t))| d \mathbf{x} .
$$

With (3.3),

and

$$
\forall r \in\left[0, \frac{\alpha}{2}\right], \forall t \in[0, \ell] \quad r \gamma(t)<\frac{1}{2}
$$

This yields

$$
\forall(t, r, \theta) \in D \quad|r(1-r \cos (\theta) \gamma(t))|=r(1-r \cos (\theta) \gamma(t))
$$

$$
\begin{aligned}
\|p\|_{H^{1}(\Omega)}^{2}= & \underbrace{\iiint_{\mathbf{x} \in D}\left(|p|^{2} \circ \Phi\right) r(1-r \cos (\theta) \gamma(t)) d \mathbf{x}}_{I_{1}} \\
& +\underbrace{\iiint_{\mathbf{x} \in D}\left(|\nabla p|^{2} \circ \Phi\right) r(1-r \cos (\theta) \gamma(t)) d \mathbf{x}}_{I_{2}} .
\end{aligned}
$$

Let us split $D$ as $D=D^{+} \cup D^{-}$with

$$
\left.D^{+}=D \cap\right] 0, \ell[\times] 0, \frac{\alpha}{2}\left[\times(]-\frac{\pi}{2}, \frac{\pi}{2}[), D^{-}=D \backslash D^{+}\right.
$$

so that $\cos \theta \geq 0$ on $D^{+}$and $\cos \theta \leq 0$ on $D^{-}$. As $(p \circ \Phi)(t, r, \theta)=(p \circ \Phi)(t, r,-\theta)$, it comes

$$
\begin{aligned}
I_{1} & =\iiint_{\mathbf{x} \in D^{+}}\left(|p|^{2} \circ \Phi\right) r(1-r \cos (\theta) \gamma(t)) d \mathbf{x}+\iiint_{\mathbf{x} \in D^{-}}\left(|p|^{2} \circ \Phi\right) r(1-r \cos (\theta) \gamma(t)) d \mathbf{x}, \\
& =\iiint_{\mathbf{x} \in D^{+}}\left(|p|^{2} \circ \Phi\right) r(1-r \cos (\theta) \gamma(t)) d \mathbf{x}+\iiint_{\mathbf{x} \in D^{+}}\left(|p|^{2} \circ \Phi\right) r(1+r \cos (\theta) \gamma(t)) d \mathbf{x}, \\
& =2 \iiint_{(t, r, \theta) \in D^{+}}\left(|p|^{2} \circ \Phi\right) r d r d t d \theta=\iiint_{(t, r, \theta) \in D}\left(|p|^{2} \circ \Phi\right) r d r d t d \theta .
\end{aligned}
$$


The computation of $I_{2}$ is different because $|(\nabla p \circ \Phi)(t, r, \theta)|=|(\nabla p \circ \Phi)(t, r,-\theta)|$ may not be true. Indeed if the tube curvature is zero then a cylindrical symmetric function has a gradient whose norm is also symmetric. This is not true any longer if the tube curvature is not zero. Set $q=p \circ \Phi$ so that

$$
q(t, r, \theta)=p(F(t)+r \cos (\theta) N(t)+r \sin (\theta) B(t)) .
$$

With the differential properties of the Frenet-Serret frame we get

$$
\left\{\begin{array}{l}
\frac{\partial q}{\partial t}=\langle\nabla p \circ \Phi,(1-r \cos (\theta) \gamma) T-r \sin (\theta) \tau N+r \cos (\theta) \tau B\rangle, \\
\frac{\partial q}{\partial r}=\langle\nabla p \circ \Phi, \cos (\theta) N+\sin (\theta) B\rangle, \\
\frac{\partial q}{\partial \theta}=\langle\nabla p \circ \Phi,-r \sin (\theta) N+r \cos (\theta) B\rangle .
\end{array}\right.
$$

As $q$ is symmetric $\frac{\partial q}{\partial \theta}=0$. So

$$
\left\{\begin{aligned}
\frac{\partial q}{\partial t} & =\langle\nabla p \circ \Phi,(1-r \cos (\theta) \gamma) T\rangle \\
\frac{\partial q}{\partial r} & =\langle\nabla p \circ \Phi, \cos (\theta) N+\sin (\theta) B\rangle \\
0 & =(\langle\nabla p \circ \Phi,-r \sin (\theta) N+r \cos (\theta) B\rangle .
\end{aligned}\right.
$$

The vector $\nabla p \circ \Phi$ is always orthogonal to $-r \sin (\theta) N+r \cos (\theta) B$ : therefore it belongs to the plane spanned by $T$ and $u=\cos (\theta) N+\sin (\theta) B$. These are orthogonal vectors and Pythagorean theorem gives

$$
|\nabla p|^{2} \circ \Phi=|\nabla p \circ \Phi|^{2}=\frac{1}{(1-r \cos (\theta) \gamma)^{2}}\left(\frac{\partial q}{\partial t}\right)^{2}+\left(\frac{\partial q}{\partial r}\right)^{2} .
$$

With the above result $I_{2}$ writes

$$
\begin{aligned}
I_{2} & =\iiint_{\mathbf{x} \in D}\left(\frac{1}{(1-r \cos (\theta) \gamma)^{2}}\left(\frac{\partial q}{\partial t}\right)^{2}+\left(\frac{\partial q}{\partial r}\right)^{2}\right) r(1-r \cos (\theta) \gamma) d \mathbf{x} \\
& =\underbrace{\iiint_{\mathbf{x} \in D} \frac{r}{1-r \cos (\theta) \gamma}\left(\frac{\partial q}{\partial t}\right)^{2} d \mathbf{x}}_{I_{3}}+\underbrace{\iiint_{\mathbf{x} \in D} r(1-r \cos (\theta) \gamma)\left(\frac{\partial q}{\partial r}\right)^{2} d}_{I_{4}} .
\end{aligned}
$$

With $D=D^{+} \cup D^{-}$, we get

$$
\begin{aligned}
I_{3} & =\iiint_{(t, r, \theta) \in D^{+}} r\left(\frac{1}{1-r \cos (\theta) \gamma}+\frac{1}{1+r \cos (\theta) \gamma}\right)\left(\frac{\partial q}{\partial t}\right)^{2} d r d t d \theta \\
& =\iiint_{(t, r, \theta) \in D} \frac{r}{1-r^{2}(\cos (\theta))^{2} \gamma^{2}}\left(\frac{\partial q}{\partial t}\right)^{2} d r d t d \theta .
\end{aligned}
$$

Similarly

$$
\begin{aligned}
I_{4} & =\iiint_{(t, r, \theta) \in D+} r(1-r \cos (\theta) \gamma+1+r \cos (\theta) \gamma)\left(\frac{\partial q}{\partial t}\right)^{2} d r d t d \theta \\
& =\iiint_{(t, r, \theta) \in D} r\left(\frac{\partial q}{\partial r}\right)^{2} d r d t d \theta
\end{aligned}
$$

Finally

$$
I_{2}=\iiint_{(t, r, \theta) \in D}\left[\omega\left(\frac{\partial q}{\partial t}\right)^{2}+\left(\frac{\partial q}{\partial r}\right)^{2}+\left(\frac{\partial q}{\partial \theta}\right)^{2}\right] r d r d t d \theta
$$


Using (4.4) we obtain (with $q=p \circ \Phi$ )

$$
I_{2}=\iiint_{(t, r, \theta) \in D}\left|\mathbb{A}_{\omega} \nabla q\right|^{2} r d r d t d \theta=\iiint_{(t, r, \theta) \in D}\left(|\mathbb{A} \nabla p|^{2} \circ \Phi\right) r d r d t d \theta .
$$

Eventually,

$$
\|p\|_{H^{1}(\Omega)}^{2}=\iiint_{(t, r, \theta) \in D}\left[\left(\left|\mathbb{A}_{\omega} \nabla p\right|^{2}+p^{2}\right) \circ \Phi\right] r d r d t d \theta
$$

and

$$
\|p\|_{H^{1}(\Omega)}^{2}=\iiint_{T_{\alpha}^{\star}}\left|\mathbb{A}_{\omega} \nabla(\Psi(p))\right|^{2}+(\Psi(p))^{2} d x .
$$

This means that $\|p\|_{H^{1}(\Omega)}^{2}=\|\Psi(p)\|_{H_{\Omega}^{1}}^{2}$. The application $\Psi$ is isometric from $\mathcal{F}_{\alpha}$ to $\mathcal{F}_{\alpha}^{\star}$. Equality $\mathcal{E}_{\varepsilon}(p)=\mathcal{E}_{\varepsilon}^{\star}(\Psi(p))$ can be proved with the same arguments.

We just proved

Theorem 4.2. Problems $\left(\mathcal{Q}_{\varepsilon, \alpha}\right)$ and $\left(\mathcal{Q}_{\varepsilon, \alpha}^{\star}\right)$ are equivalent. More precisely $p$ is the unique solution to $\left(\mathcal{Q}_{\varepsilon, \alpha}\right)$ if and only if $\Psi(p)$ is the unique solution to $\left(\mathcal{Q}_{\varepsilon, \alpha}^{\star}\right)$.

4.3. Solution regularity. We end this section by giving regularity properties of $\left(\mathcal{Q}_{\varepsilon, \alpha}\right)$ solution. For this, we first show that the solution of the problem $\left(\mathcal{Q}_{\varepsilon, \alpha}^{\star}\right)$ is solution of a more general problem. Then we will use Theorem 4.2 to conclude. Consider

$$
\min _{q \in H_{0}^{1}\left(T_{\alpha}^{\star}\right)} \mathcal{E}_{\varepsilon}^{\star}(q) .
$$

It is now classical to see that if $\beta \leq \varepsilon$ then problem $\left(\mathcal{Q}_{\varepsilon, \alpha}^{\star \star}\right)$ has a unique solution.

Theorem 4.3. Assume $\beta \leq \varepsilon$ and let $p^{\star}$ be the solution of $\left(\mathcal{Q}_{\varepsilon, \alpha}^{\star \star}\right)$. Then $p^{\star} \in \mathcal{C}^{\infty}\left(T_{\alpha}\right) \cap \mathcal{F}_{\alpha}^{\star}$.

Proof. We first prove that any solution $p$ of $\left(\mathcal{Q}_{\varepsilon, \alpha}^{\star \star}\right)$ belongs to $\mathcal{C}^{\infty}\left(T_{\alpha}^{\star}\right)$. The first order optimality condition gives

$$
\forall \varphi \in H_{0}^{1}\left(T_{\alpha}^{\star}\right) \quad \int_{T_{\alpha}^{\star}}\left(9 \beta \varepsilon \nabla p \nabla \varphi+f_{\beta, \varepsilon}(p) \varphi\right) d x=0,
$$

where $f_{\beta, \varepsilon}$ is given by (3.8). Then

$$
9 \beta \varepsilon \Delta p=f_{\beta, \varepsilon}(p) \text { on } T_{\alpha}^{\star},
$$

in the sense of distributions. As $T_{\alpha}^{\star}$ is a smooth open subset of $\mathbb{R}^{3}$, then $H_{0}^{1}\left(T_{\alpha}\right) \subset$ $L^{6}\left(T_{\alpha}\right)$ ([2] for example). This implies that $f_{\beta, \varepsilon}(p) \in L^{2}\left(T_{\alpha}^{\star}\right)$. Thanks to the ellipticity of the Laplacian operator we deduce that $p$ is $C^{\infty}$.

Let us show now that $p^{\star} \in \mathcal{F}_{\alpha}^{\star}$. We use a symmetrization technique. Let $H$ be an hyperplane including $\Gamma^{\star}$, $\Pi$ the orthogonal symmetry with respect to $H$ and set $\tilde{p}:=p \circ \Pi$. As problem $\left(\mathcal{Q}_{\varepsilon, \alpha}^{\star \star}\right)$ is invariant by the rotations of axis $\Gamma^{\star}$, the function $\tilde{p}$ is solution of the problem as well. By uniqueness, $\tilde{p}=p^{\star}$. this proves that $p^{\star}$ has a cylindrical symmetry of axis $\Gamma^{\star}$.

We may now conclude:

Theorem 4.4. If $\beta \leq \varepsilon$, the unique solution to $\bar{p}$ de $\left(\mathcal{Q}_{\varepsilon, \alpha}\right)$ belongs to $\mathcal{C}^{2}\left(T_{\alpha}\right)$.

Proof. Theorem 4.3 tells that the unique solution $p^{\star}$ of $\left(\mathcal{Q}_{\varepsilon, \alpha}^{\star}\right)$ belongs to $\mathcal{C}^{\infty}\left(T_{\alpha}^{\star}\right)$. In addition, $\bar{p}=\Psi^{-1}\left(p^{\star}\right)$ is the unique solution of $\left(\mathcal{Q}_{\varepsilon, \alpha}\right)$. As $\Psi^{-1}\left(p^{\star}\right)$ as the same regularity as $\Phi$, we deduce that $\bar{p}$ belongs to $\mathcal{C}^{2}\left(T_{\alpha}\right)$ since $\Phi$ is $\mathcal{C}^{2}$. 


\section{Conclusion}

The model we have presented allows to consider thin structures segmentation via a geometrical prior. The tuning of parameters allows to separate noise from small tubes. However, we are only able to perform a study on the low-level problem. In addition, the study of the low-level solution shows that the (full) model we consider is too rigid to follow the tubes. Therefore, we have to add an anisotropic term, namely a moving vector field that makes it more flexible. This is detailed in [25].

Last but not least, we actually perform numerical simulations and different tests with respect to the parameters $\alpha, \beta$ and $\varepsilon$. This will be reported in a near future.

\section{REFERENCES}

[1] David R. Adams. Capacity and the obstacle problem. Appl. Math. Optim., 8(1):39-57, 1982.

[2] Robert Adams and John Fournier. Sobolev spaces. Number 140 in Pure and Applied Mathematics Serie. Academic Press, 2003.

[3] Luigi Ambrosio, Nicola Fusco, and Diego Pallara. Functions of bounded variation and free discontinuity problems. Oxford mathematical monographs,. Oxford University Press, 2000.

[4] Luigi Ambrosio and Vincenzo Maria Tortorelli. Approximation of functionnals depending on jumps by elliptic functionnals via gamma-convergence. Communications on Pure and Applied Mathematics, XLIII:999-1036, 1990.

[5] Jung-Ha An, Paul Bigeleisen, and Steve Damelin. Identification of nerves in ultrasound scans using modified mumford-shah model and prior information. In Proceedings of the World Congress on Engineering and Computer Science 2011 Vol I WCECS 2011, October 19-21, 2011, San Francisco,, 2011.

[6] Hedy Attouch, Giuseppe Buttazzo, and Gérard Michaille. Variationnal Analysis in Sobolev and BV spaces. SIAM, 2005.

[7] Gilles Aubert, Jean-François Aujol, and Laure Blanc-Féraud. Detecting codimension-two objects in an image with ginsburg-landau models. 5254, INRIA, 2004.

[8] Gilles Aubert, Laure Blanc-Féraud, and Daniele Graziani. Analysis of a new variational model to restore point-like and curve-like singularities in imaging. Appl. Math. Optim., 67(1):73-96, 2013.

[9] Gilles Aubert and Daniele Graziani. Variational approximation for detecting point-like target problems. ESAIM Control Optim. Calc. Var., 17(4):909-930, 2011.

[10] Gilles Aubert and Daniele Graziani. A relaxation result for an inhomogeneous functional preserving point-like and curve-like singularities in image processing. Internat. J. Math., 23(11):1250120, 14, 2012.

[11] Gilles Aubert and Pierrre Kornprobst. Mathematical Problems in Image Processing, Partial Differential Equations and the Calculus of Variations, volume 147 of Applied Mathematical Sciences. Springer Verlag, 2006.

[12] Guy David. Mumford-Shah minimizers on thin plates. Calc. Var. Partial Differential Equations, 27(2):203-232, 2006.

[13] Gilles A. Francfort, Nam Q. Le, and Sylvia Serfaty. Critical points of Ambrosio-Tortorelli converge to critical points of Mumford-Shah in the one-dimensional Dirichlet case. ESAIM Control Optim. Calc. Var., 15(3):576-598, 2009.

[14] Daniele Graziani, Laure Blanc-Féraud, and Gilles Aubert. A formal $\Gamma$-convergence approach for the detection of points in 2-D images. SIAM J. Imaging Sci., 3(3):578-594, 2010.

[15] Cemil Kirbas and Francis Quek. A review of vessel extraction techniques and algorithms. Journal ACM Computing Surveys (CSUR), 36(2):81-121, June 2004.

[16] Esther Klann, Ronny Ramlau, and Wolfgang Ring. A Mumford-Shah level-set approach for the inversion and segmentation of SPECT/CT data. Inverse Probl. Imaging, 5(1):137-166, 2011.

[17] David Lesage, Elsa D Angelini, Isabelle Bloch, and Gareth Funka-Lea. A review of 3d vessel lumen segmentation techniques: Models, features and extraction schemes. Medical Image Analysis, 13:819845, 2009.

[18] Sasan Mahmoodi. Edge detection filter based on Mumford-Shah Green function. SIAM J. Imaging Sci., 5(1):343-365, 2012.

[19] Luciano Modica. The gradient theory of phase transitions and the minimal interface criterion. Archive for Rational Mechanics and Analysis, 98:123-142, 1987. 
[20] David Mumford and Jayant Shah. Optimal approximations by piecewise smooth functions and associated variationnal problems. Communications on Pure and Applied Mathematics, XLVII(5):577-685, 1989.

[21] Mickael Péchaud, Gabriel Peyré, and Renaud Keriven. Extraction of vessels networks over an orientation domain. 2008.

[22] Ronny Ramlau and Wolfgang Ring. Regularization of ill-posed Mumford-Shah models with perimeter penalization. Inverse Problems, 26(11):115001, 25, 2010.

[23] Youssef Rouchdy and Laurent Cohen. Image segmentation by geodesic voting. application to the extraction of tree structures from confocal microscope images. In ICPR 2008. 19th International Conference on Pattern Recognition, pages 1-5, 2008.

[24] David Vicente. A $\gamma$ - convergence result for a mumford-shah modified model. Preprint, December 2013.

[25] David Vicente. $\gamma$-convergence of anisotropic vectorial functionals adapted to detection of thin tubes. Technical report, http://hal.archives-ouvertes.fr/hal-01006255, June 2014.

University of Orléans, Laboratory MAPMO, CNRS, UMR 7349, Fédération Denis PoisSON, FR 2964, BÂtiment de Mathématiques, BP 6759, 45067 Orléans Cedex 2, France, MAiTINE.BERGOUNIOUX, DAVID.VICENTE@UNIV-ORLEANS.FR 